\title{
Complexity of Two-Level Logic Minimization
}

\author{
Christopher Umans, Tiziano Villa, and Alberto L. Sangiovanni-Vincentelli, Fellow, IEEE
}

\begin{abstract}
The complexity of two-level logic minimization is a topic of interest to both computer-aided design (CAD) specialists and computer science theoreticians. In the logic synthesis community, two-level logic minimization forms the foundation for more complex optimization procedures that have significant real-world impact. At the same time, the computational complexity of two-level logic minimization has posed challenges since the beginning of the field in the 1960s; indeed, some central questions have been resolved only within the last few years, and others remain open. This recent activity has classified some logic optimization problems of high practical relevance, such as finding the minimal sum-of-products (SOP) form and maximal term expansion and reduction. This paper surveys progress in the field with self-contained expositions of fundamental early results, an account of the recent advances, and some new classifications. It includes an introduction to the relevant concepts and terminology from computational complexity, as well a discussion of the major remaining open problems in the complexity of logic minimization.
\end{abstract}

Index Terms-Computational complexity, logic design, logic minimization, two-level logic.

\section{INTRODUCTION}

$\mathbf{C}$ OMPUTER-AIDED design (CAD) specialists [14], [32] and computer science theoreticians [26] alike investigated the computational complexity of logic minimization problems. The motivation lies both in their practical importance for design automation [3], [4], [28] and in their paradigmatic nature in the landscape of computational complexity classes.

Even though logic synthesis has grown increasingly sophisticated, building complex optimization scenarios that span from regular fabrics of restricted depth [5], [18] up to multilevel and multivalued logic realizations [8], two-level logic minimization [good old time programmable logic arrays (PLAs)] retains a central role as a key procedure in more complex logic optimization packages. Indeed, the search for better algorithms for two-level logic minimization has been pursued relentlessly, delivering practically efficient tools from ESPRESSO [21] to ESPRESSO-SIGNATURE [17] to SCHERZO [6]. SCHERZO uses

Manuscript received October 4, 2004; revised March 10, 2005. The work of C. Umans was supported by National Science Foundation (NSF) under Grant CCF-0346991. The work of T. Villa was supported by Project for Advanced Research of Architecture and Design of Electronic Systems (PARADES). The work of A. L. Sangiovanni-Vincentelli was supported by the Center for Hybrid and Embedded Software Systems (CHESS) under Contract NSF ITR Grant CCR-0225610. This paper was recommended by Associate Editor L. Stok.

C. Umans is with the Department of Computer Science, California Institute of Technology, Pasadena, CA 91125 USA (e-mail: umans@ @ cs.caltech.edu).

T. Villa is with Department of Electrical, Industrial and Mechanical Engineering (DIEGM), Universita' di Udine, Udine 33100, Italy and also with PARADES, Rome 00186, Italy (e-mail: villa@ parades.rm.cnr.it).

A. L. Sangiovanni-Vincentelli is with the Department of Electrical Engineering and Computer Sciences (EECS), University of California at Berkeley, Berkeley, CA 94720 USA and also with PARADES, Rome 00186, Italy (e-mail: alberto@parades.rm.cnr.it).

Digital Object Identifier 10.1109/TCAD.2005.855944 two graph-based data structures to represent Boolean functions, namely, binary decision diagrams (BDDs, called OBDDs when they are reduced and ordered) and zero-suppressed decision diagrams (ZDDs); BDDs and ZDDs have the advantage that there is no linear relation between their sizes and the number of elements of the sets that they represent. More recently, SATESPRESSO [22] was proposed, which is a re-implementation of ESPRESSO based on a SAT solver as the underlying engine.

Therefore, an in-depth investigation of two-level logic minimization has a special place in studying the complexity of logic synthesis problems. The history of classifying the complexity of two-level logic minimization accompanies the field of computational complexity from its beginnings in the 1960s [10] to now. This history extends to a result of only a few years ago settling a conjecture open for more than 20 years, namely, that the following problem is $\Sigma_{2}^{p}$-complete [30], [31]: "given a sum-of-product (SOP) form of a logic function, is there an equivalent SOP form with at most a given number of terms?" This result spawned the complexity classification of the main optimization problems routinely solved by software packages like ESPRESSO, such as maximal term expansion and reduction. With this recent activity, important theoretical achievements are linked with state-of-the-art exact or heuristic algorithmic practice.

Relevant material is scattered in various sources and some key older items are hard to find and/or inaccurate in their original version. This paper presents the progress of the field with a self-contained exposition that sets the record straight, states precisely the results, and introduces the most valuable proof techniques. The CAD practitioner should gain an updated understanding of the complexity of relevant fundamental problems, while the theorist will find a gentle survey of this literature.

The remainder of this paper is organized as follows. Section II introduces basic definitions related to logic functions. Section III gives a brief introduction to the relevant complexity classes and to their canonical complete problems. Section IV discusses the relation between two-level minimization and covering problems by introducing Gimpel's reduction that shows the $N P$-completeness of two versions of this problem. Section V reports a revised version of Masek's proof that SOP minimization is $N P$-complete when the input is given by the full truth table of a completely specified function. Section VI discusses the complexity of two-level minimization and various subproblems used in major logic minimization suites (i.e., ESPRESSO) when the input is given as an SOP. Many of these problems turn out to be $\Sigma_{2}^{p}$-complete. Section VII reviews past achievements and major open problems.

\section{BACKGROUND ON BOOLEAN FUNCTIONS}

Let $f$ be a Boolean function. An implicant of $f$ is a conjunction $C$ of literals that implies $f$. For a conjunction $C$, we 
denote by $\operatorname{lit}(C)$ the set of literals appearing in $C$. In this notation, if $C_{1}$ and $C_{2}$ are conjunctions with $\operatorname{lit}\left(C_{1}\right) \subseteq \operatorname{lit}\left(C_{2}\right)$ and $C_{1}$ is an implicant of $f$, then $C_{2}$ also is an implicant of $f$.

A conjunction $C$ covers those assignments with no variable set in contradiction to its setting in $C$. We write $C_{1} \subseteq C_{2}$ to mean that the minterms covered by $C_{1}$ are a subset of the minterms covered by $C_{2}$. Note that $C_{1} \subseteq C_{2}$ is equivalent to $\operatorname{lit}\left(C_{1}\right) \supseteq \operatorname{lit}\left(C_{2}\right)$.

An implicant $C$ is a prime implicant (or just a "prime") if the only conjunction $C^{\prime} \supseteq C$ that is an implicant of $f$ is $C$ itself. An essential prime implicant is a prime implicant that covers some assignment that is covered by no other prime implicant.

An implicant is also called a product or term, and a disjunction of products of literals is called an SOP. We write $\phi=\vee_{i \in I} t_{i}$ to mean the SOP formula $\phi$ with terms $t_{i}$ for $i \in I$. Formula $\phi$ is equivalent to formula $\phi^{\prime}$, written $\phi \equiv \phi^{\prime}$, if and only if (iff) the two SOPs cover exactly the same assignments (i.e., the functions computed by $\phi$ and $\phi^{\prime}$ are the same).

\section{INTRODUCTION TO COMPUTATIONAL COMPLEXITY}

In this section, we introduce the main concepts from the computational complexity needed in the paper. We refer to [2], [9], and [19] as standard references.

For the purposes of this paper, a computational problem is formalized as a decision problem. Given as input a string (the instance), the solution to a decision problem is either "yes" or "no." The set of strings whose answer is "yes" completely specifies the problem and is sometimes called the language associated with the decision problem.

If $\Sigma$ is a finite alphabet and $L \subseteq \Sigma^{*}$ is a language, we define the complement language, denoted $\bar{L}$, as the set of strings that are not in $L$ (in other words, $\bar{L}=\Sigma^{*}-L$ ). In most reasonable schemes for encoding instances of real-world problems as strings, some strings do not correspond to valid encodings of any instance. For this reason, we often abuse notation slightly and use $\bar{L}$ to refer to only those strings in $\Sigma^{*}-L$ that are valid encodings of some instance. This is standard and does not affect any of the results we cite or prove.

Example 3.1: CNF-SAT is the problem of deciding if a given Boolean expression in conjunctive normal form (CNF) has a satisfying assignment. Given a reasonable rule to encode CNF expressions, the language CNF-SAT will contain all strings in $\Sigma^{*}$ that encode CNF expressions that are satisfiable. The complement problem $\overline{\text { CNF-SAT }}$ is the problem of deciding if the given $\mathrm{CNF}$ is unsatisfiable. The language $\overline{\mathrm{CNF}-\mathrm{SAT}}$ contains all strings that encode CNF expressions that are unsatisfiable. As noted above, strings in $\Sigma^{*}$ that do not encode any CNF expression are neither in CNF-SAT nor in CNF-SAT.

Computational complexity studies the computational resources required to solve problems. Formally, an algorithm for a decision problem is a Turing Machine that accepts exactly those strings in the associated language and rejects those not in the language. ${ }^{1} \mathrm{We}$ are primarily interested in the worst-case running time of such an algorithm, as measured in steps taken by

\footnotetext{
${ }^{1}$ Some problems are undecidable and possess no such algorithm, but they are not of interest here.
}

the Turing Machine. This measure is polynomially related to the running time of all other commonly used computational mechanisms (for instance, a $\mathrm{C}$ program running on a Von Neumann computer).

A complexity class is a set of languages. As we are interested in running time, an important class is $P$, the set of languages possessing algorithms that run in time that is a polynomial in the length of the input. Another important class of languages is $N P$, the set of languages possessing algorithms that run in nondeterministic polynomial time. For this paper, we will use the following alternate equivalent definition.

Definition 3.1: A language $L$ is in the class $N P$ iff there is another language $R$ in the class $P$ and an integer $k$ for which

$$
L=\left\{x: \exists y,|y| \leq|x|^{k},(x, y) \in R\right\} .
$$

For example, CNF-SAT is a language in $N P$. This is true because we can define the language $R$ in $P$ to consist of those pairs $(\phi, A)$ for which $\phi$ is a CNF formula, and $A$ is a satisfying assignment for $\phi$. We see that CNF-SAT $=\{\phi: \exists A,(\phi, A) \in$ $R\}$, which shows that CNF-SAT is in $N P$. It can be seen from the above definition that $N P$ are exactly those languages whose "yes" instances possess succinct witnesses that can be verified in polynomial time.

To understand the importance of $N P$ in studying the running time required for various problems, we must describe the central notions of reductions and completeness in computational complexity. A reduction from language $A$ to language $B$ is a transformation $T$ that maps "yes" instances of $A$ to "yes" instances of $B$ and "no" instances of $A$ to "no" instances of $B$. Formally, $T$ is a function from $A$ to $B$ that satisfies $x \in A \Leftrightarrow$ $T(x) \in B$. We require our reductions $T$ to be computable in polynomial time. Intuitively, a reduction $T$ from $A$ to $B$ that runs in polynomial time implies that $B$ is at least as hard as $A$.

Let $\mathcal{C}$ be a complexity class. A language $L$ is $\mathcal{C}$-hard if every language in $\mathcal{C}$ reduces to $L$ in polynomial time. Such a language may be regarded as "at least as hard" as any language in $\mathcal{C}$. A language $L$ that is $\mathcal{C}$-hard and also in the class $\mathcal{C}$ is called $\mathcal{C}$-complete. Such a language may be regarded as the "hardest" language in $\mathcal{C}$. Complete languages are very useful because they allow one to reason about an abstract complexity class by studying a concrete natural computational problem. Perhaps the most well known example of a complete problem is CNF-SAT.

Theorem 3.1 [Cook (see [9])]: CNF-SAT is NP-complete.

The importance of the class $N P$ in studying the time complexity of problems stems from 1) the fact that many natural problems are known to be $N P$-complete, and 2) the widely believed assumption that $P \neq N P$. If any $N P$-hard problem is in $P$ then $P=N P$, and so showing that a problem is $N P$-hard amounts to a proof (subject to the above assumption) that the problem does not have any polynomial-time algorithm.

We now describe some complexity classes beyond $P$ and $N P$ that we will need to correctly classify some of the problems we encounter in this paper.

In general, for a complexity class $\mathcal{C}$, we can define the complement class, denoted $c o \mathcal{C}$, to be the set of all complements of languages in $\mathcal{C}$, i.e., $c o \mathcal{C}=\{L: \bar{L} \in \mathcal{C}\}$. The complement of $P$ is just $P$ again, but the complement of $N P$ is the class 
$\operatorname{coNP}$, which is believed to be different from $N P$. Using the fact that $P$ is closed under complement, and Definition 3.1, we arrive at the following.

Definition 3.2: A language $L$ is in the class $\operatorname{coN} P$ iff there is another language $R$ in class $P$ and an integer $k$ for which

$$
L=\left\{x: \forall y,|y| \leq|x|^{k},(x, y) \in R\right\}
$$

The canonical $c o N P$-complete language is SOP-VALIDITY (also called DNF-TAUTOLOGY). This language consists of all strings encoding SOP expressions that are satisfiable by all truth assignments, or "valid." From Theorem 3.1, we know that $\overline{\mathrm{CNF}-S A T}$ is $c o N P$-complete, and a simple reduction (mapping CNF formula $\phi$ to SOP expression $\neg \phi$ ) then shows that SOP-VALIDITY is $c o N P$-complete.

Both NP-complete and coNP-complete problems are "hard" in the sense that they cannot have polynomial-time algorithms under the assumption $P \neq N P$. Still, it is useful to maintain the distinction, because in practice heuristics attacking $N P$ problems can stop once they find a witness; and this positive feature cannot be exploited for a $c o N P$-complete problem (assuming $N P \neq \operatorname{coNP}$ ).

A more esoteric class that we will need is the class $D P$, which is defined in terms of $N P$ and $\operatorname{coNP}$.

Definition 3.3: The class $D P$ is the set of languages $L$ that can be expressed as

$$
L=\{x: x \in A \text { and } x \in B\}
$$

where $A$ is a language in $N P$ and $B$ is a language in $\operatorname{coNP}$.

The canonical complete language for $D P$ is the language SAT-UNSAT, which consists of all pairs $\left(\phi_{1}, \phi_{2}\right)$, where $\phi_{1}$ is a satisfiable CNF expression and $\phi_{2}$ is an unsatisfiable CNF expression. Note that $D P$ contains all of $N P$ (because given a language $L$ in $N P$, it can be seen to lie in $D P$ by taking $A=L$ and $B=\Sigma^{\star}$ ) and $D P$ also contains all of the $\operatorname{coNP}$ (because given a language $L$ in $c o N P$, we can take $A=\Sigma^{\star}$ and $B=L$ ). Thus, a problem that is $D P$-hard is both $N P$-hard and $c o N P$ hard (although the converse is not necessarily true).

\section{A. Oracles and the Polynomial Hierarchy}

We say that a Turing Machine is equipped with an oracle $L$ when it has available a subroutine that charges one unit of computation to answer whether a given string is in $L$. For example, a Turing Machine equipped with a CNF-SAT oracle can in a single step determine whether a CNF expression generated in the course of its computation is satisfiable by "querying" its oracle. We define complexity classes involving oracles using a standard shorthand: if $\mathcal{C}$ and $\mathcal{B}$ are complexity classes, then $\mathcal{C}^{\mathcal{B}}$ is the class of languages decided by a machine of the type that defines $\mathcal{C}$, augmented with oracle language in $\mathcal{B}$. This is not a precise definition, but the meaning should be clear for the classes we apply this to.

An example of an oracle complexity class is $P^{N P}$. This class includes all languages decidable by a Turing Machine running in polynomial time that is equipped with a CNF-SAT oracle since CNF-SAT is in $N P{ }^{2}$ An example of a language in $P^{N P}$ is the language consisting of all $m$-tuples of CNF expressions for which an odd number of them are satisfiable. Given such an instance $\left(\phi_{1}, \phi_{2}, \ldots, \phi_{m}\right)$, we can make $m$ queries to the CNF-SAT oracle to determine exactly which of the $m$ expressions are satisfiable and accept if the number of satisfiable expressions is odd.

A refinement of $P^{N P}$ is the class $P_{\|}^{N P}$, in which the queries to the oracle are required to be nonadaptive. That is, no query depends on the outcome of previous queries, and so the computation can always be organized as follows: first compute in polynomial time a set of polynomially many oracle queries, then perform the queries "in parallel," and finally decide whether to accept or reject the input with a polynomial-time computation on the input and the outcomes of the parallel queries. The example above in fact lies in $P_{\|}^{N P}$. Clearly, $P_{\|}^{N P}$ is contained in $P^{N P}$, and we observe that $D P, N P$, and $c o N P$ are all contained in $P_{\|}^{N P}$. Complexity theorists believe that all of these classes are distinct.

Just as it is meaningful to augment a polynomial-time deterministic Turing Machine with an oracle, we can also augment a polynomial-time nondeterministic Turing Machine with an oracle. This gives rise to an infinite hierarchy of complexity classes collectively called the polynomial hierarchy $(\mathrm{PH})$. We describe the levels of the PH below using the shorthand for oracle classes and follow that with an alternate (formal) definition that generalizes Definition 3.1, i.e.,

$$
\begin{array}{ll}
\Sigma_{0}^{P}=P & \Pi_{0}^{P}=P \\
\Sigma_{1}^{P}=N P & \Pi_{1}^{P}=c o N P \\
\Sigma_{2}^{P}=N P^{N P} & \Pi_{2}^{P}=c o N P^{N P} \\
\quad \vdots \quad & \\
\Sigma_{i}^{P}=N P^{\Sigma_{i-1}^{P}} & \Pi_{i}^{P}=\operatorname{coN} P^{\Sigma_{i-1}^{P}}
\end{array}
$$

Definition 3.4: A language $L$ is in the class $\Sigma_{i}^{P}$ iff there is another language $R$ in the class $P$ and an integer $k$ for which

$$
\begin{aligned}
L=\left\{x:\left(\exists y_{1}\right)\left(\forall y_{2}\right)\left(\exists y_{3}\right)\right. & \cdots\left(Q y_{i}\right),\left|y_{i}\right| \leq|x|^{k} \\
& \text { for all } \left.i,\left[\left(x, y_{1}, y_{2}, \ldots, y_{i}\right) \in R\right]\right\}
\end{aligned}
$$

where the sequence of quantifiers alternates, ending with $Q=\exists$ if $i$ is odd or $Q=\forall$ if $i$ is even.

Definition 3.5: A language $L$ is in the class $\Pi_{i}^{P}$ iff there is another language $R$ in the class $P$ and an integer $k$ for which

$$
\begin{aligned}
L=\left\{x:\left(\forall y_{1}\right)\left(\exists y_{2}\right)\left(\forall y_{3}\right)\right. & \cdots\left(Q y_{i}\right),\left|y_{i}\right| \leq|x|^{k} \\
& \text { for all } \left.i,\left[\left(x, y_{1}, y_{2}, \ldots, y_{i}\right) \in R\right]\right\}
\end{aligned}
$$

\footnotetext{
${ }^{2}$ In fact, $P^{\mathrm{CNF}-\mathrm{SAT}}=P^{N P}$, because for any Turing Machine running in polynomial time that is equipped with any language $L \in N P$ as an oracle, we can build another Turing Machine running in polynomial time equipped with a CNF-SAT oracle that decides the same language. The new Turing Machine simply applies the polynomial-time reduction from $L$ to CNF-SAT prior to making each of its oracle calls
} 
where the sequence of quantifiers alternates, ending with $Q=\forall$ if $i$ is odd or $Q=\exists$ if $i$ is even.

A few facts can be immediately gleaned from these definitions. First, $\Pi_{i}^{P}=c o \Sigma_{i}^{P}$ for all $i$. Second, for each $i, \Sigma_{i}^{P}$ contains $\Pi_{i-1}^{P}$ and $\Sigma_{i-1}^{P}$. In particular, $\Sigma_{2}^{P}$ contains both $N P$ and $\operatorname{coN} P$, and it is not much more difficult to show that it contains in addition all of the other classes we have considered, namely $D P, P_{\|}^{N P}$, and $P^{N P}$. The most important class in the PH for this paper will indeed be $\Sigma_{2}^{p}$; an example of a natural problem in this class follows.

Example 3.2: The language EQUIVALENT FORMULAS consists of those pairs $(\phi, k)$ where $\phi$ is a Boolean expression for which there exists an equivalent Boolean expression $\phi^{\prime}$ of length at most $k$.

This language is in $\Sigma_{2}^{P}$ because we can define a language $R$ that lies in $P$ as follows: $R$ accepts those tuples $\left((\phi, k), \phi^{\prime}, A\right)$ for which $\phi$ and $\phi^{\prime}$ are Boolean formulas, $\phi^{\prime}$ has length of at most $k$, and $\phi$ agrees with $\phi^{\prime}$ on assignment $A$. We then see that

\section{$(\phi, k) \in$ EQUIVALENT FORMULAS}

$$
\Leftrightarrow\left(\exists \phi^{\prime}\right)(\forall A)\left[\left((\phi, k), \phi^{\prime}, A\right) \in R\right] .
$$

It is not known whether EQUIVALENT FORMULAS is $\Sigma_{2}^{p}$ complete or not, although many people believe that it is. Some natural problems have been shown to be complete for various levels of the PH above the first level (see the surveys [23], [24]), although not nearly the same number as are known to be $N P$ complete.

The canonical complete problems for the $\mathrm{PH}$ are "quantified satisfiability" problems. The instances of these problems are Boolean expressions $\phi$ built on a set of Boolean variables $\cup_{i} X_{i}$, where $X_{i}=\left\{x_{i, j}: 1 \leq j \leq m_{i}\right\}$ for positive integers $m_{i}$. The language $k$-QBF consists of those expressions for which

$$
\left(\exists X_{1}\right)\left(\forall X_{2}\right) \cdots\left(Q X_{k}\right)\left[\phi\left(X_{1}, X_{2}, \ldots, X_{k}\right)\right]
$$

where the sequence of quantifiers alternates, ending with $Q=\exists$ if $k$ is odd or $Q=\forall$ if $k$ is even. Here, " $\left(\exists X_{i}\right)$ " is to be read as "there exists an assignment of values to the variables $x_{i, 1}, \ldots, x_{i, m_{i}}$ ", and " $\left.\forall X_{i}\right)$ " is to be read as "for all assignments of values to the variables $x_{i, 1}, \ldots, x_{i, m_{i}}$ "

Theorem 3.2 (see [19]): For all $k \geq 1$, the problem $k$-QBF is $\Sigma_{k}^{p}$-complete.

\section{Covering and Minterm Minimization Problems}

We begin our examination of the complexity of two-level logic minimization by considering several variants of the problem of finding a minimum SOP representation of a specified Boolean function, described below.

\section{INCOMPLETE TRUTH TABLE MIN SOP}

INSTANCE: Onset and offset of incompletely specified function $f$ (i.e., disjoint sets $A, B \subseteq\{0,1\}^{n}$ ) and a positive integer $k$.

QUESTION: Is there an SOP representation of $f$ (i.e., an SOP formula $\phi$ for which $x \in A \Rightarrow \phi(x)=1$ and $x \in$ $B \Rightarrow \phi(x)=0$ ) with at most $k$ terms?
FULL TRUTH TABLE MIN SOP

INSTANCE: Onset and offset of completely specified function $f$ (i.e., disjoint sets $A, B \subseteq\{0,1\}^{n}$ with $A \cup$ $\left.B=\{0,1\}^{n}\right)$ and a positive integer $k$.

QUESTION: Is there an SOP representation of $f$ (i.e., an SOP formula $\phi$ for which $x \in A \Rightarrow \phi(x)=1$ and $x \in$ $B \Rightarrow \phi(x)=0$ ) with at most $k$ terms?

ONSET TRUTH TABLE MIN SOP

INSTANCE: Onset of completely specified function $f$ (i.e., a set $A \subseteq\{0,1\}^{n}$ ) and a positive integer $k$.

QUESTION: Is there an SOP representation of $f$ (i.e., an SOP formula $\phi$ for which $\phi(x)=1 \Leftrightarrow x \in A$ ) with at most $k$ terms?

All three of these problems are in $N P$ because given a "candidate" SOP $\phi$ with at most $k$ terms, one can determine whether it is a representation of $f$ in polynomial time in the size of the instance. For the first and second problems, this simply requires evaluating $f$ at all of the points in $A$ and $B$. For the third problem, we can check that $\phi$ is a representation of $f$ as follows: we explicitly construct the set $\{x: \phi(x)=1\}$ by adding the minterms covered by each term in $\phi$ one at a time and only once. If at any point our set size exceeds $|A|$, we know that $\phi$ is not a representation of $f$; otherwise, if at the end our set equals $A$, we know that $\phi$ is indeed a representation of $f$.

In fact, all three of these problems are $N P$-complete. We will give proofs of this fact for the first and the third problem in this section. The $N P$-completeness proof for the problem FULL TRUTH TABLE MIN SOP is contained in Section V. We first discuss the "covering problems" that play an important role in the reductions.

\section{A. Incomplete Truth Table MIN SOP}

A classical exact procedure for finding the minimum SOP representation of a Boolean function $f$ is due to QuineMcCluskey [16]. This procedure first computes all of the prime implicants of $f$ and then finds a minimum cardinality subset of these prime implicants that cover all of the minterms of $f$.

The first part of this procedure requires polynomial time in the size of the onset of $f$ [27] (and so is efficient for any of the three variants of TRUTH TABLE MIN SOP that we consider above). The second part of the Quine-McCluskey procedure is a special case of the well-known $N P$-complete problem below.

\section{MINIMUM COVER}

INSTANCE: Collection $C$ of subsets of a finite set $S$ and a positive integer $k \leq|C|$.

QUESTION: Does $C$ contain a cover for $S$ of size at most $k$; i.e., is there a subset of $C^{\prime} \subseteq C$ with $\left|C^{\prime}\right| \leq k$ such that every element of $S$ belongs to at least one member of $C^{\prime}$ ?

MINIMUM COVER has been shown to be $N P$-complete in Karp's seminal paper [13]. It is also well known that the problem remains $N P$-complete even when we restrict the subsets in $C$ to all having a size of exactly three [9].

Now it might be the case that when we restrict to the subproblem of MINIMUM COVER consisting of only those instances arising from the Quine-McCluskey procedure, the problem 
becomes tractable. A couple of papers [10], [20] investigated this question and gave transformations that show that even this subproblem remains $N P$-complete. We summarize here their main results.

It will be convenient to represent covering problems as matrices, or covering tables. The elements of $S$ index the rows and the columns are indexed by the subsets of $C$. Entry $(i, j)$ is 1 if subset $j$ contains element $i$, in which case column $j$ is said to cover row $i$.

Definition 4.1: Let $X$ and $Y$ be finite sets. A function $A$ : $X \times Y \rightarrow\{0,1\}$ is a covering table iff

- $\forall x \in X \exists y \in Y: A(x, y)=1$

- $\forall y \in Y \exists x \in X: A(x, y)=1$.

A subset $Z \subseteq Y$ is called a cover of $X$ if $\forall x \in X \exists z \in Z$ : $A(x, z)=1$.

As noted, a Boolean function $f$, possibly incompletely specified, gives rise to a covering table. Adapting terminology from [20], we denote by $P I(f)$ the prime implicants of $f$ and by $A_{f^{-1}(1), P I(f)}$ the covering table whose rows are the minterms of $f$ and whose columns are the prime implicants of $f$, called also the Minterm-Prime (MP) table of $f$.

Despite the fact that an MP table seems to possess additional structure, it turns out that every covering table can arise as the MP table of an incompletely specified function.

Theorem 4.1: Let $A: X \times Y \rightarrow\{0,1\}$ be a covering problem such that $A$ has no equal rows, and let $k=|Y|$. Then, there is an incompletely specified Boolean function $f: D \rightarrow\{0,1\}$ with $D \subseteq\{0,1\}^{k}$ for which $A_{f^{-1}(1), P I(f)}$ equals $A$ (possibly after renaming rows and columns).

Proof: We define the positive monotone ${ }^{3}$ function $f$ as follows. The offset contains a single point: the all zeros point. The onset contains one minterm for each row of $A$ : the minterm whose coordinates are exactly that row of $A$.

It is easy to verify that the primes of $f$ are exactly those implicants with a single 1 , with all zeros raised to "-". 4 Thus, $|P I(f)|=k$. Moreover, the $j$ th column of $A$ equals the column of $A_{f^{-1}, P I(f)}$ labeled with the prime $p$ that has a 1 in the $j$ th position. This is because for each $i$, the $i$ th row of $A$ corresponds to a minterm of $f$ that is covered by $p$ iff entry $A(i, j)=1$.

Example 4.1: Consider the following covering table $A$ with $X=\{1,2,3\}$ and $Y=\{1,2,3\}$

\begin{tabular}{|l||l|l|l|}
\hline & 1 & 2 & 3 \\
\hline \hline 1 & 0 & 1 & 1 \\
\hline 2 & 1 & 0 & 1 \\
\hline 3 & 1 & 1 & 0 \\
\hline
\end{tabular}

The above transformation gives the incompletely specified function $f$ that has $f^{-1}(0)=\{000\}$ and $f^{-1}(1)=$

\footnotetext{
${ }^{3}$ A function $f$ is positive monotone if $x<y$ implies $f(x)<f(y)$.

${ }^{4}$ In this section, we use an alternative notation for implicants: a string of $0 \mathrm{~s}$, $1 \mathrm{~s}$, and -s. A 0 (resp. 1) in location $i$ means that literal $\bar{x}_{i}$ (resp. $x_{i}$ ) appears in the implicant.
}

$\{011,101,110\}$. The primes of $f$ are $1--,-1-$, and --1 , and $A_{f^{-1}(1), P I(f)}$ is

\begin{tabular}{|c||c|c|c|}
\hline & $1--$ & $-1-$ & --1 \\
\hline \hline 011 & 0 & 1 & 1 \\
\hline 101 & 1 & 0 & 1 \\
\hline 110 & 1 & 1 & 0 \\
\hline
\end{tabular}

The following problem formalizes the second step in the Quine-McCluskey algorithm.

MINTERM-PRIME MINIMUM COVER

InStAnCE: Minterm-Prime table $A: X \times Y \rightarrow\{0,1\}$ and a positive integer $k \leq|Y|$.

QUESTIONS: Does $A$ contain a cover of size at most $k$; i.e., is there a cover $Z \subseteq Y$ with $|Z| \leq k$ ?

Theorem 4.1 can be interpreted as a reduction from an instance of MINIMUM COVER to an instance of MINTERMPRIME MINIMUM COVER, which gives the following.

Theorem 4.2: MINTERM-PRIME MINIMUM COVER is $N P$ complete.

Also using Theorem 4.1, we have a reduction from MINIMUM COVER to INCOMPLETE TRUTH TABLE MIN SOP, giving the following.

Theorem 4.3: INCOMPLETE TRUTH TABLE MIN SOP is $N P$ complete.

This can be interpreted to mean that there are in fact no "better ways" to solve the problem of INCOMPLETE TRUTH TABLE MIN SOP than Quine-McCluskey—any alternative approach must also be $N P$-hard.

\section{B. Onset Truth Table MIN SOP}

The transformation in Theorem 4.1 cannot be used directly to prove that ONSET TRUTH TABLE MIN SOP Or FULL TRUTH TABLE MIN SOP is $N P$-complete. The reason is that it produces a function $f$ whose number of variables $k$ is equal to the number of columns of the original covering table $A$. A completely specified function consistent with $f$ might have exponentially many minterms and would certainly have an exponentially large truth table. Thus, a reduction to ONSET TRUTH TABLE MIN SOP Or FULL TRUTH TABLE MIN SOP based directly on Theorem 4.1 would not be a polynomial-time reduction.

However, a different transformation than the one in Theorem 4.1 will allow us to prove the $N P$-hardness of ONSET TRUTH TABLE MIN SOP. The same authors [10], [20] describe how to produce a completely specified function that in a certain sense still represents the original covering table.

Theorem 4.4: Let $A: X \times Y \rightarrow\{0,1\}$ be a covering problem such that $A$ has no row that dominates another ${ }^{5}$ and let $n=|X|$. Then there is a completely specified function $g$ : $\{0,1\}^{n+2} \rightarrow\{0,1\}$ whose MP table equals $A$ after removal of

\footnotetext{
${ }^{5} \mathrm{~A}$ row dominates another if the $1 \mathrm{~s}$ in the first row are a superset of the $1 \mathrm{~s}$ in the second. We can remove dominant rows in polynomial time. The resulting covering problem is equivalent to the initial one.
} 
the essential prime implicants and of the minterms they cover (possibly after renaming rows and columns).

Proof: We construct $g$ in several steps. We first construct a function $g^{\prime}$ whose variables are $z_{1}, z_{2}, \ldots, z_{n}$.

- For each $i \in\{1, \ldots,|X|\}$, define minterm $q_{i}=z_{1}, \ldots$, $z_{i-1} \bar{z}_{i} z_{i+1}, \ldots, z_{n}$.

- For each $j \in\{1, \ldots,|Y|\}$, define $Q_{j}=\prod_{i: A(i, j)=0} z_{i}$.

- Let $p_{1}, p_{2}, \ldots, p_{m}$ be an enumeration of all minterms covered by the $Q_{j}$ for some $j$ that are not among the $q_{i}$. We may assume that the first $\ell$ of these minterms have an even number of negated variables, and the remaining $m-\ell$ have an odd number of negated variables.

- We define $g$ whose variables are $z_{1}, z_{2}, \ldots, z_{n+2}$ as

$$
\begin{aligned}
g=\sum_{i=1}^{n} q_{i} z_{n+1} z_{n+2} & +\sum_{i=1}^{m} p_{i} z_{n+1} z_{n+2} \\
& +\sum_{i=1}^{\ell} p_{i} z_{n+1} \bar{z}_{n+2}+\sum_{i=\ell+1}^{m} p_{i} \bar{z}_{n+1} z_{n+2} .
\end{aligned}
$$

We can verify that the prime implicants of $g$ are exactly.

- $Q_{j} z_{n+1} z_{n+2}$ for each $j$

- $p_{i} z_{n+1}$ for $i=1,2, \ldots, \ell$

- $p_{i} z_{n+2}$ for $i=\ell+1, \ell+2, \ldots, m$.

The last two of these sets of primes are actually essential prime implicants. After removing these essential prime implicants and the minterms that they cover, we are left with minterms $q_{i} z_{n+1} z_{n+2}$, and prime implicants $Q_{j} z_{n+1} z_{n+2}$. Notice that $A(i, j)=1$ iff $q_{i} z_{n+1} z_{n+2}$ is covered by $Q_{j} z_{n+1} z_{n+2}$, and hence the MP table of $g$ is equivalent to $A$ after removal of the essential prime implicants and the minterms they cover.

Example 4.2: Starting with the covering table $A$ from Example 4.1 , we have $q_{1}=\bar{z}_{1} z_{2} z_{3}, q_{2}=z_{1} \bar{z}_{2} z_{3}, q_{3}=z_{1} z_{2} \bar{z}_{3}$ and $Q_{1}=z_{1}, Q_{2}=z_{2}, Q_{3}=z_{3}$.

There are four minterms covered by $Q_{j}$ that are not among the $q_{i}$. They are $p_{1}=z_{1} z_{2} z_{3}, p_{2}=z_{1} \bar{z}_{2} \bar{z}_{3}, p_{3}=\bar{z}_{1} z_{2} \bar{z}_{3}$, and $p_{4}=\bar{z}_{1} \bar{z}_{2} z_{3}$. In this example, all of the $p_{i}$ have an even number of negated variables, so we obtain

$$
g=\sum_{i=1}^{3} q_{i} z_{4} z_{5}+\sum_{i=1}^{4} p_{i} z_{4} z_{5}+\sum_{i=1}^{4} p_{i} z_{4} \bar{z}_{5} .
$$

The primes of $g$ are $z_{1} z_{4} z_{5}, z_{2} z_{4} z_{5}, z_{3} z_{4} z_{5}, z_{1} z_{2} z_{3} z_{4}$, $z_{1} \bar{z}_{2} \bar{z}_{3} z_{4}, \bar{z}_{1} z_{2} \bar{z}_{3} z_{4}$, and $\bar{z}_{1} \bar{z}_{2} z_{3} z_{4}$, of which all but the first three are essential. After removing the essential prime implicants and the minterms they cover, we are left with minterms $\bar{z}_{1} z_{2} z_{3} z_{4} z_{5}, z_{1} \bar{z}_{2} z_{3} z_{4} z_{5}, z_{1} z_{2} \bar{z}_{3} z_{4} z_{5}$ and primes $z_{1} z_{4} z_{5}, z_{2} z_{4} z_{5}, z_{3} z_{4} z_{5}$. This portion of $g$ 's MP table is shown as

\begin{tabular}{|c||c|c|c|}
\hline & $1--11$ & $-1-11$ & --111 \\
\hline \hline 01111 & 0 & 1 & 1 \\
\hline 10111 & 1 & 0 & 1 \\
\hline 11011 & 1 & 1 & 0 \\
\hline
\end{tabular}

which can be seen to equal the covering table $A$.
Czort [7] described how to use this construction to prove that ONSET TRUTH TABLE MIN SOP is NP-complete.

Theorem 4.5: ONSET TRUTH TABLE MIN SOP is NPcomplete.

Proof: We have already argued that ONSET TRUTH TABLE MIN SOP is in $N P$. We reduce from the variant of MINIMUM COVER in which every set has a size of exactly three (which remains $N P$-complete). An instance of this problem gives a covering table $A$ in which each column has exactly three ones. Our reduction outputs the function $g$ coming from the transformation in Theorem 4.4.

We critically use the fact that $A$ has only three ones per column to ensure that the onset of $g$ has size polynomial in the size of $A$. Using the terminology of the proof of Theorem 4.4, the onset of $g$ has $n+2 m$ points. Each $Q_{j}$ has exactly $n-3$ variables, and so it can cover at most $2^{3}$ points. Thus, $m$ is at most $|Y| 2^{3}$. So the onset of $g$ has at most $|X|+16|Y|$ points, which is polynomial in the size of $A$.

There are exactly $m$ essential prime implicants of $g$, and the remaining MP table is equivalent to $A$. Thus, $g$ has an SOP representation with at most $k$ terms iff $A$ has a cover of size at most $k-m$. This completes the reduction.

\section{Full Truth Table MiN-SOP is NP-Complete}

Masek [15] showed that the problem of finding a minimum SOP representation is NP-complete even when the input is given as a fully specified truth table. For this problem, an input of size $n$ specifies a function of only $\log n$ variables. An efficient algorithm for this problem (one that runs in polynomial time in the size of the input) is therefore allowed exponential time in the number of variables. It is further evidence of the hardness of this problem that even under these favorable circumstances an efficient solution is unlikely.

The heart of Masek's reduction ${ }^{6}$ is a method for "embedding" any Boolean circuit $C$ of size $n$ into a $O(\log n)$ dimension Boolean hypercube, whose subcubes of dimension one are its vertices or nodes. Roughly speaking, the gates of the circuit are embedded by setting a cluster of adjacent vertices to one; specified vertices in this cluster are the input and output vertices. These clusters are the gadgets used in the reduction. Wires between an input vertex and an output vertex belonging to different gadgets are embedded by setting the hypercube vertices along a path between them to one. The gadgets and wires are separated sufficiently so that no product in an SOP cover of the resulting function can cover vertices belonging to different gadgets or distinct wires.

By an interpretation described in more detail below, every SOP cover assigns truth values (TRUE or FALSE) to the input and output vertices according to how they are covered. Each gadget is designed so that it is covered most efficiently (i.e., by the fewest products) when it "computes" the intended function of its inputs. Similarly, the wires are designed so that they are covered most efficiently when they "transmit" a consistent truth value from their source to their destination. The final gadget in the embedding has a single input and is most efficiently covered 


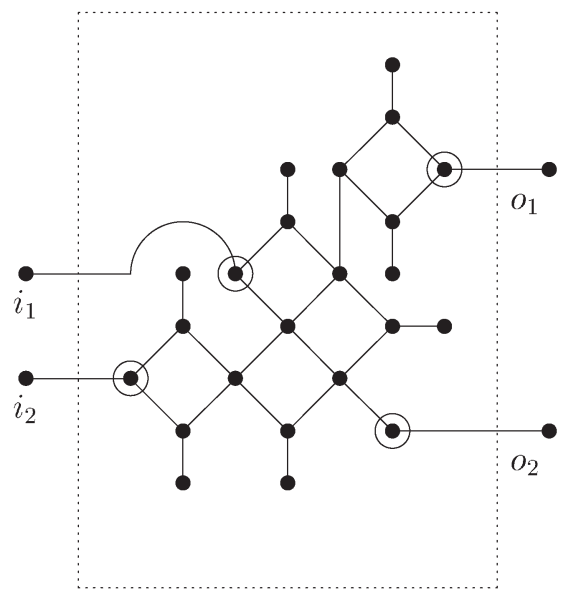

Fig. 1. AND/OR gadget.

when that input has value TRUE. By connecting the output of the embedded circuit to this gadget, we ensure that the function on $O(\log n)$ variables defined by this embedding has a small SOP cover iff the circuit $C$ is satisfiable.

We will describe the desired embedding with the aid of an adjacency diagram, whose vertices correspond to nodes of the hypercube that are set to one and whose edges connect exactly those pairs of nodes that differ in exactly 1 bit. As a final step, we will argue that the adjacency diagram we produce is realizable in a hypercube of $O(\log n)$ dimensions.

As mentioned, the basic components of the embedding are gadgets with distinguished I/O nodes and wires between them. In our embedding, every $\mathrm{I} / \mathrm{O}$ node will be adjacent to a node belonging to a wire. Given a SOP cover, we say that an I/O node is TRUE if it is covered by a product that also covers the adjacent wire node, and FALSE otherwise.

In counting the number of terms in an SOP cover for our embedding, we would like to be able to count the terms that cover each component separately, and then sum them to get the total number. This is not quite possible because some product may simultaneously cover nodes in a gadget and an adjacent wire. To avoid double counting this product, we adopt the following convention: for a product covering both an I/O node and the adjacent wire node, we charge $1 / 2$ to the gadget and $1 / 2$ to the wire. This simple accounting trick will simplify the exposition that follows significantly.

\section{A. AND/OR Gadget}

The AND/OR gadget has two inputs, $i_{1}$ and $i_{2}$, and two outputs, which "compute" the AND and OR of the two input truth values, respectively. It has the adjacency structure shown in Fig. 1.

Lemma 5.1: Given an SOP cover $C$, let $x$ and $y$ be the truth values assigned to the I/O nodes labeled $i_{1}$ and $i_{2}$ in the figure, respectively. Then $C$ covers the AND/OR gadget with 11 terms if it assigns truth values $x \wedge y$ and $x \vee y$ to the $\mathrm{I} / \mathrm{O}$ nodes labeled $o_{1}$ and $o_{2}$, respectively. Otherwise, $C$ covers the AND/OR gadget with more than 11 terms.

Proof: The full proof (by case analysis) can be found in the Appendix.

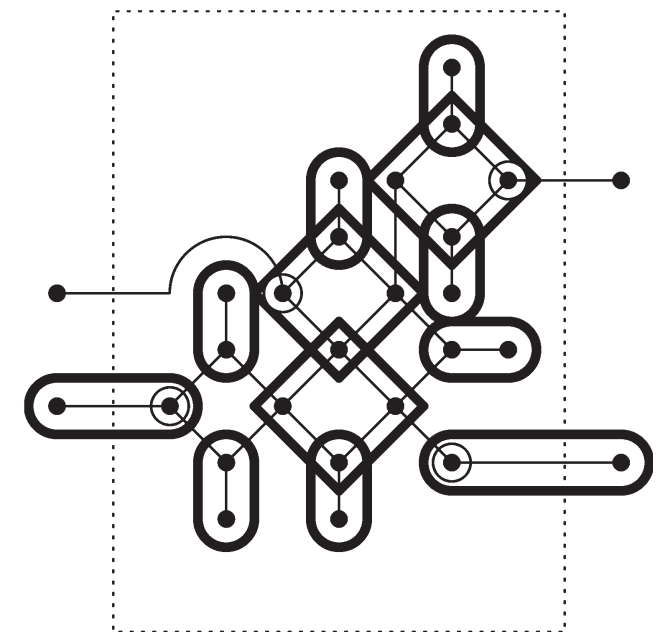

Fig. 2. Cover of AND/OR gadget.

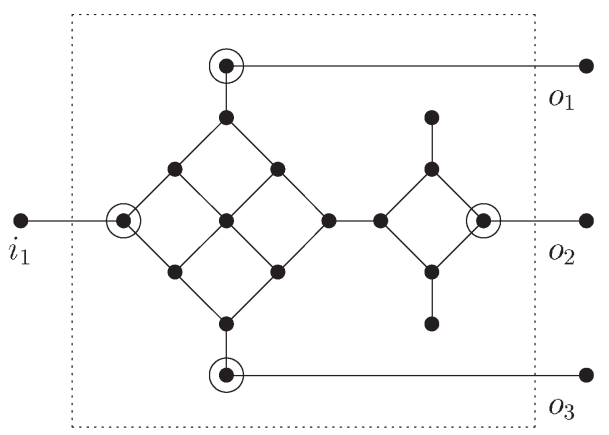

Fig. 3. GENERATOR gadget.

Fig. 2 shows one possible cover of an AND/OR gadget. In this example, $x$ is FALSE and $y$ is TRUE. Note that the two terms that extend outside the dotted line each contribute $1 / 2$ to the overall sum of 11 , according to our accounting convention.

\section{B. GENERATOR Gadget}

The GENERATOR gadget has a single input $i_{1}$ and three outputs, which "generate" three copies of the input truth value. It has the adjacency structure shown in Fig. 3.

Lemma 5.2: Given an SOP cover $C$, let $x$ be the truth value assigned to the I/O node labeled $i_{1}$ in the figure. Then $C$ covers the GENERATOR gadget with seven terms if it assigns truth value $x$ to the $\mathrm{I} / \mathrm{O}$ nodes labeled $o_{1}, o_{2}$, and $o_{3}$. Otherwise, $C$ covers the GENERATOR gadget with more than seven terms.

Proof: The full proof (by case analysis) can be found in the Appendix.

Fig. 4 shows two possible covers of a GENERATOR gadget, each using seven terms. In the first cover, $x$ is TRUE; in the second cover, $x$ is FALSE.

\section{WIRE Gadget}

The WIRE gadget has no I/O nodes of its own; it is used to connect two I/O nodes belonging to GENERATOR or AND/OR gadgets. A WIRE gadget is simply a path between two I/O 

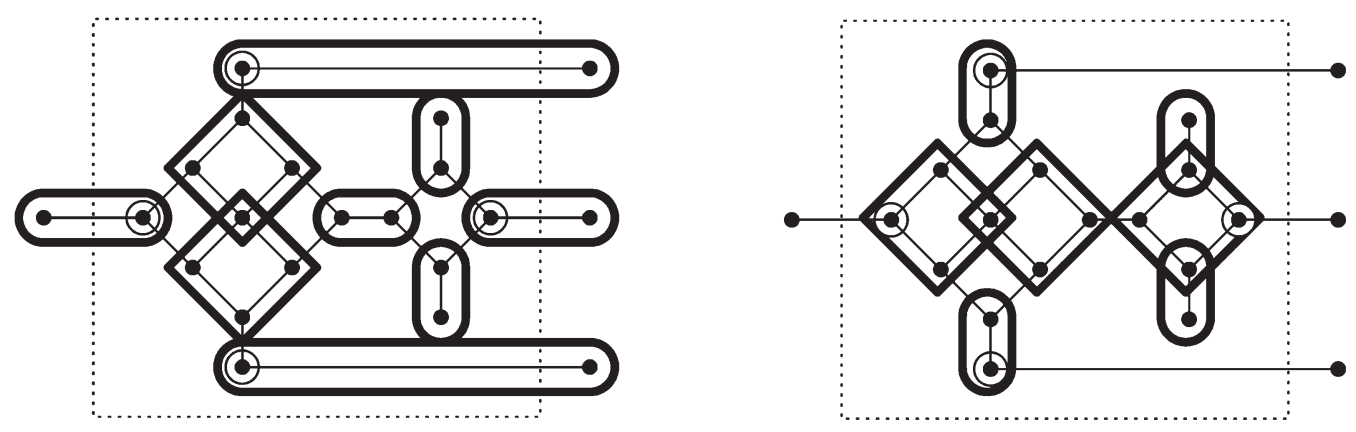

Fig. 4. Covers of GENERATOR gadget.
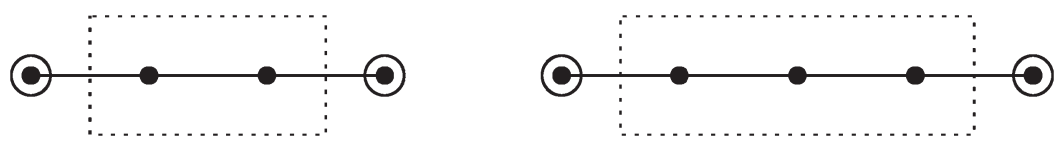

Fig. 5. WIRE gadget.
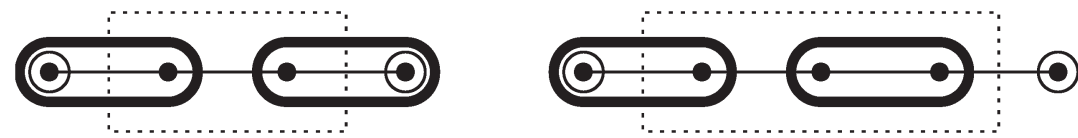

Fig. 6. Covers of WIRE gadget.

nodes. The length of the wire is the number of nodes in the path, not counting the I/O nodes at either end. WIRE gadgets of length 2 and 3 are shown in Fig. 5.

Lemma 5.3: An SOP cover $C$ covers a WIRE gadget of length $k$ with at most $k / 2$ terms if either

- the wire has even length and $C$ assigns the same truth values to the $\mathrm{I} / \mathrm{O}$ nodes connected by the wire, or

- the wire has odd length and $C$ assigns opposite truth values to the I/O nodes connected by the wire.

Otherwise, $C$ covers the WIRE gadget with more than $k / 2$ terms.

Proof: It is clear that only terms covering one or two points can cover points belonging to the WIRE gadget; optimal covers in the two cases are then easy to determine by inspection.

Fig. 6 shows covers of the two WIRE gadgets. The first cover uses one term and assigns TRUE to the I/O nodes connected by the wire. The second cover uses $3 / 2$ terms and assigns TRUE to the I/O node on the left and FALSE to the I/O node on the right.

We remark that it is common in some of the more complicated proofs of $N P$-completeness to have "gadgets" such as these that play a crucial role in reduction.

\section{Building the Adjacency Diagram}

We now describe how to attach these gadgets together to "simulate" a given Boolean formula $f\left(x_{1}, x_{2}, \ldots, x_{n}\right)$. We assume that $f$ has fan-in 2, fan-out 1 AND and OR gates, and WLOG that all the negations occur at the leaves. In fact, we will simulate both $f$ and its complement; the added symmetry is needed, for example, because we do not have a separate AND gadget at our disposal, but only a combined AND/OR gadget.

The final adjacency diagram will be coverable by at most $N$ terms iff $f$ is satisfiable, where $N$ is the sum over all gadgets in the diagram of the optimal cover numbers given in the previous three lemmas.

We first create a set of "variables" sufficient to supply the variable values at the leaves of the formula. For each $i$, let $n_{i}$ be the number of occurrences of the variable $x_{i}$ as a leaf of the $f$. Because we are simulating $f$ and its complement, we need to supply $2 n_{i}$ occurrences of $x_{i}$; however, we also know that there will be an equal number of positive and negative occurrences of $x_{i}$, which will make the construction easier. If $n_{i}=1$, a single odd-length wire will supply a positive and negative occurrence of $x_{i}$, one at either end of the wire.

If $n_{i}=2$, we use two GENERATOR gadgets. We connect the input I/O nodes of these two GENERATOR gadgets with an odd length wire, and we connect the third outputs of these two generator gadgets with an odd length wire. The remaining four outputs (two belonging to each of the GENERATOR gadgets) provide two copies each of positive and negative occurrences of $x_{i}$. For $n_{i}>2$, we repeat this process: pick an output supplying a positive occurrence of $x_{i}$ and connect a fresh GENERATOR gadget to it with an even length wire, and similarly, pick an output supplying a negative occurrence of $x_{i}$ and connect a fresh GENERATOR gadget to it with an even length wire. Finally, connect the third outputs of each of these two fresh GENERATOR gadgets with an odd length wire. After connecting $2\left(n_{i}-1\right)$ GENERATOR gadgets in this fashion, we have, altogether, $n_{i} \mathrm{I} / \mathrm{O}$ nodes that supply positive occurrences of $x_{i}$ and $n_{i} \mathrm{I} / \mathrm{O}$ nodes that supply negative occurrences of $x_{i}$. Fig. 7 shows a cover of the adjacency diagram of an example of generators for $n_{i}=3$; the generator gadgets supply three copies of variable $x_{i}$ and three copies of its complement. In the pictured cover, $x_{i}$ is TRUE for all copies and $\bar{x}_{i}$ is FALSE for all copies.

We can now construct the "gates" that simulate the gates of $f$. For each AND or OR gate $g$ in the lowest level of the formula, 


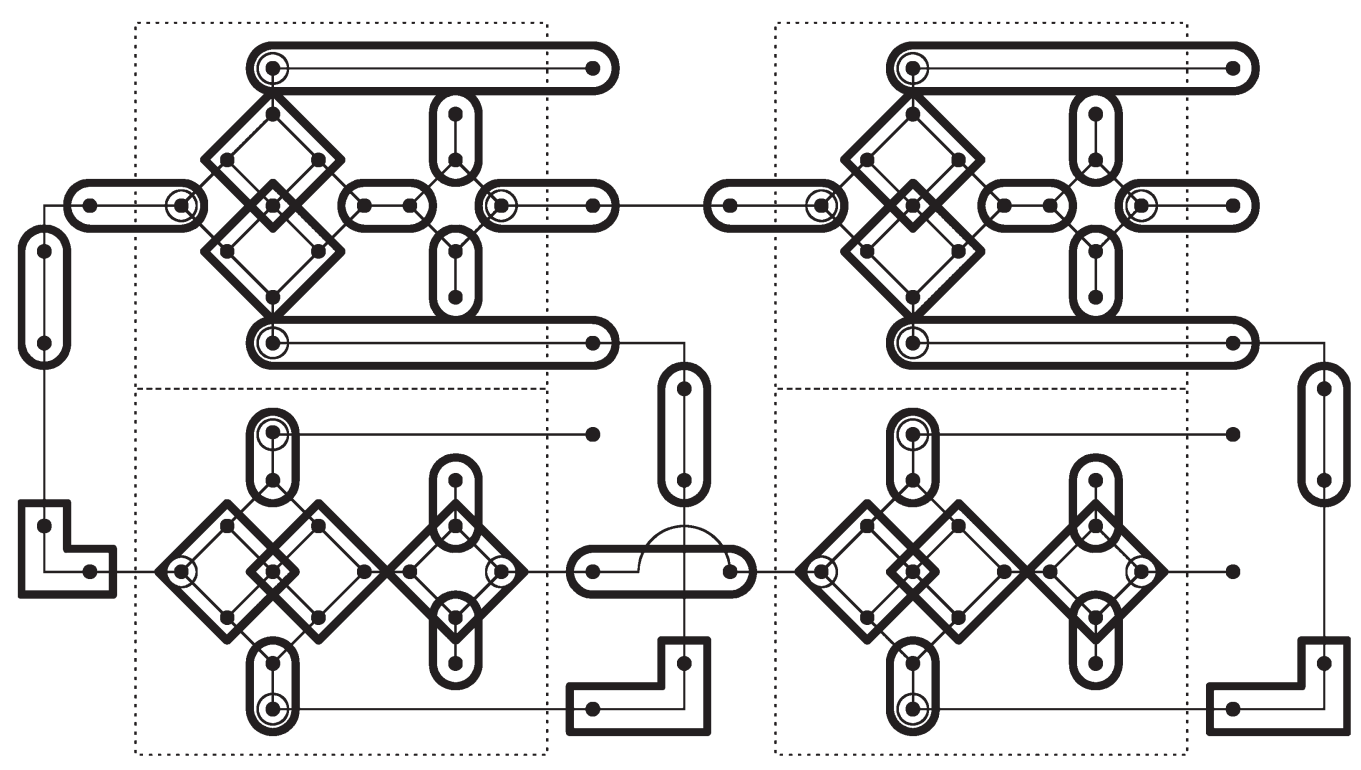

Fig. 7. Cover of generators supplying three copies of variable $x_{i}$ (and three copies of its complement).

we have an AND/OR gadget. Let $x_{i}^{a}$ and $x_{j}^{b}$ (where $a, b \in\{0,1\}$ ) be the two literals that are inputs to gate $g$ in the formula $f$. We attach the two input I/O nodes of the AND/OR gadget to I/O nodes supplying the literals $x_{i}^{a}$ and $x_{j}^{b}$ with two even length wires. We also add an additional AND/OR gadget that will compute the complement of $g$. We attach the two input I/O nodes of this AND/OR gadget to I/O nodes supplying the literals $x_{i}^{1-a}$ and $x_{j}^{1-b}$ with two even length wires. Finally, if $g$ was an AND gate, we attach the second output (the "OR" output) of the first AND/OR gadget to the first output (the "AND" output) of the second AND/OR gadget with an odd length wire. If $g$ was an OR gate, we attach the first output (the "AND" output) of the first AND/OR gadget to the second output (the "OR" output) of the second AND/OR gadget with an odd length wire. The remaining two outputs supply the value $g\left(x_{i}^{a}, x_{j}^{b}\right)$ and its complement $\neg g\left(x_{i}^{a}, x_{j}^{b}\right)$.

Now, we move on to the next level of gates, using the outputs from the previous level as the inputs for the current level, and so on. The final gate's output is attached to an odd length wire, and the complementary output is attached to an even length wire. Note that this odd length wire (say, with length $k$ ) can be covered with $k / 2$ terms if it assigns TRUE to the single I/O node to which it is attached, and it requires more than $k / 2$ terms otherwise. Similarly, the even length wire (say, with length $k^{\prime}$ ) can be covered with $k^{\prime} / 2$ terms if it assigns FALSE to the single $\mathrm{I} / \mathrm{O}$ node to which it is attached; otherwise, it requires more than $k^{\prime} / 2$ terms.

\section{E. Realizing the Adjacency Diagram}

In the previous section, we have built an adjacency diagram with $m=O(|f|)$ gadgets. These gadgets have certain points that play the role of input nodes and certain points that play the role of output nodes. In the above construction, we have used odd-length wires to connect input nodes to input nodes, and also to connect output nodes to output nodes. We have used even length wires to connect output nodes to input nodes.
In this section, we describe how to map the points of the adjacency diagram from the previous section into points of a hypercube of dimension $O(\log m)$. This mapping will realize the adjacency diagram: a pair of nodes in the adjacency diagram will be mapped to two nodes in the hypercube that differ in exactly 1 bit iff there is an edge in the adjacency diagram between those two nodes. In order to construct this realization, we need to allow ourselves the freedom to choose wire lengths that may differ from the ones in the adjacency diagram; however, the odd/even parity of their lengths (which is what matters) will be preserved.

As an example of a realization of the adjacency diagrams of the AND/OR and GENERATOR gadgets in a hypercube of dimension six, see Fig. 8. This embedding will play an important role in the "global" realization momentarily.

We first describe how to map all of the nodes belonging to AND/OR and GENERATOR gadgets into the hypercube. In the following description, the weight of a bit string is the number of ones in it; the parity of a bit string is "even" if it has even weight and "odd" otherwise. For each of the AND/OR and GENERATOR gadgets, we assign a unique even parity ID string of length $2 \ell$ (the parameter $\ell$ will be specified momentarily), and for each of the WIRE gadgets we assign a unique even parity ID string of length $4 \ell$ and weight $2 \ell$. We ensure that every pair of distinct WIRE gadget ID strings differs in at least four positions. This can be achieved, for example, by choosing each WIRE gadget ID string to be an even parity string of length $2 \ell$ with weight $\ell$, repeated twice. If there are $m$ gadgets in the diagram (including WIRE gadgets), we need $\ell=O(\log m)$ to be able to choose the IDs as described. ${ }^{7}$

Our hypercube will have dimension $6 \ell+6$, and for convenience we routinely refer to nodes of the hypercube with threetuples whose fields have bit lengths $2 \ell, 6$ and $4 \ell$, respectively.

\footnotetext{
${ }^{7}$ From $\left(\begin{array}{c}2 \ell \\ \ell\end{array}\right)>m$ (the number of $2 \ell$ bit strings with weight $\ell$ must be at least $m$ ) and $\left(\begin{array}{c}2 \ell \\ \ell\end{array}\right)>2^{\ell}$, it suffices to take $\ell=\log _{2} m$.
} 


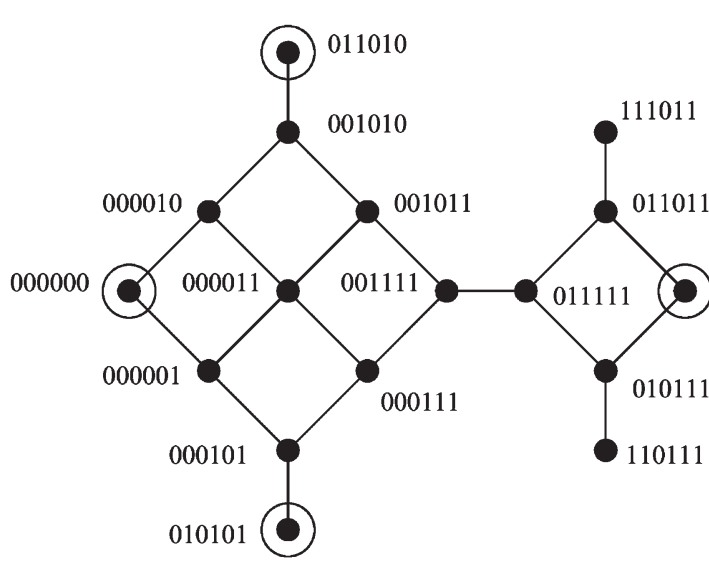

Fig. 8. Embedding of GENERATOR (left) and AND/OR (right) gadgets.

For AND/OR or GENERATOR gadget $i$ that has been assigned ID id $(i)$, we map the node labeled with the 6-bit string $x$ in Fig. 8 to the hypercube node $(\operatorname{id}(i), x, \overrightarrow{0})$ (where $\overrightarrow{0}$ refers to the $4 \ell$-bit all-zero string). The fact that this is a valid embedding so far follows from the validity of the embeddings in Fig. 8.

We now turn to the wires in the adjacency diagram. Note that, from the previous paragraph and Fig. 8, all of the input I/O nodes are embedded at hypercube nodes with even parity and all of the output I/O nodes are embedded at hypercube nodes with odd parity (our construction requires to connect input points to input points and output points to output points with odd length wires, and output points to input points with even length wires; this implies that the parity-number of ones in the encoding vectors - of the outputs must be the same and opposite to the parity of the inputs). For each wire, we will describe a sequence of adjacent points whose endpoints are the I/O nodes that the wire connects. By the above observation regarding the parity of the various I/O node labels, the length of these embedded wires will retain the required even/odd parity that they have in the adjacency diagram (even if their lengths may change).

A wire with $\operatorname{ID~} \operatorname{id}(k)$ between gadgets $i$ and $j$ with $\operatorname{IDs} \operatorname{id}(i)$ and $\operatorname{id}(j)$, respectively, is embedded as follows. Let $x$ and $y$ be the 6-bit labels of the I/O nodes we are connecting, belonging to gadget $i$ and $j$, respectively. The wire will contain the following hypercube nodes:

1) $(\operatorname{id}(i), x, \overrightarrow{0})$;

2) $(\operatorname{id}(i), x, \operatorname{id}(k))$;

3) $(\operatorname{id}(j), y, \operatorname{id}(k))$;

4) $(\operatorname{id}(j), y, \overrightarrow{0})$.

In addition, we include the hypercube nodes along an arbitrary shortest path between each of these points, changing 1 bit a time. For example, between the first and second points in the above list, we include $2 \ell-1$ additional hypercube nodes to get from $\overrightarrow{0}$ to id $(k)$.

There are two special wires in our adjacency diagram that do not terminate at I/O nodes; the odd length wire connected to the output of the final gate of the circuit and the even length wire connected to its complement output. We may take this even length wire to be a length 0 wire (and therefore we need to add

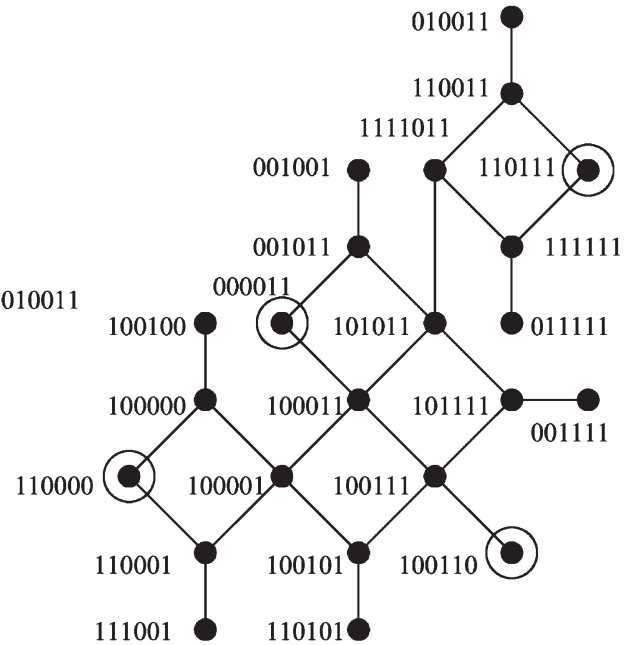

no new nodes to our embedding). For the odd-length wire (with label $\operatorname{id}(k)$ ), which is connected to some node with 6-bit label $x$ in some gadget $i$ with label id $(i)$, we add the single hypercube node $(\operatorname{id}(i), x, 00 \cdots 01)$. This forms a wire of length 1 .

The validity of the overall embedding may be verified by case analysis. The main effort involves verifying that we have not introduced any "unintended" adjacencies in our embedding (to that purpose the third field of the embedding vectors plays a role, especially to enforce that there is no spurious adjacency between points across wires).

Lemma 5.4: The embedding just described realizes the adjacency diagram of the Boolean formula $f$ in an $O(\log |f|)$ dimension Boolean hypercube.

Proof: The full proof (by case analysis) can be found in the Appendix.

Our main theorem is as follows.

Theorem 5.1: In the above embedding of $f$, let $N$ be the sum of the following

- the total length of wires divided by 2 ;

- the number of AND/OR gadgets times 11;

- the number of GENERATOR gadgets times 7 .

Then the function whose onset is exactly those hypercube nodes in the above embedding has an SOP cover of size $N$ iff the function $f$ is satisfiable.

Proof: If $f$ is satisfiable, we can have each gadget and each wire behave "as intended," and this defines a cover with exactly $N$ products using Lemmas 5.1, 5.2, and 5.3.

In the other direction, suppose we have a cover $C$ with exactly $N$ products. Then, by Lemmas 5.1, 5.2, and 5.3, every gadget must behave "as intended"-for if even a single gadget did not, the number of products of the cover would exceed $N$. We conclude that the output of the $f$ is one for some setting of its input variables. This setting is determined from the truth values $C$ defined at the input I/O nodes. The fact that $C$ optimally covers the special odd length wire extending from the final output node implies that $f$ evaluates to TRUE for this setting of the variables.

Observe that if we write down the complete truth table of the function on $6 \ell+6$ variables whose onset is exactly those 
hypercube nodes in the above embedding, it will have a length of $2^{O(\log |f|)}$, which is polynomial in $|f|$. The mapping from formula $f$ to this truth table is straightforward to compute, and thus the overall transformation can be computed in polynomial time in $|f|$. This observation together with Theorem 5.1 gives us the following.

Theorem 5.2: FULL TRUTH TABLE MIN SOP is NPcomplete.

A different reduction to show that FULL TRUTH TABLE MIN SOP is $N P$-complete (this time reducing from SET COVER) has been recently obtained by Allender et al. [1]. This proof also gives new inapproximability results for FULL TRUTH TABLE MIN SOP.

\section{SOP Minimization Problems are $\Sigma_{2}^{P}$-Complete}

In this section, we discuss logic minimization problems whose input is an SOP form, whereas in the previous formulations the input was a truth table. The prototypical such problem is MIN SOP-2-SOP asking, given an SOP formula $\phi$, what is the smallest SOP $\phi^{\prime}$ equivalent to $\phi .{ }^{8}$ Can this problem be in $N P$ ? It cannot, if one believes (as complexity theorists do) that coNP is different from NP-because MIN SOP-2-SOP can easily be seen to be $c o N P$-hard. ${ }^{9}$ In fact, MIN SOP-2-SOP exhibits the $\exists \forall$ quantifier alternation of $\Sigma_{2}^{P}$-it asks if there exists a short-SOP $\phi^{\prime}(x)$ such that for all $x, \phi^{\prime}(x)=\phi(x)$-so we might expect it to be $\Sigma_{2}^{P}$-complete. Indeed, MIN SOP-2-SOP was conjectured to be complete for $\Sigma_{2}^{P}$ in a seminal 1976 paper by Stockmeyer [26].

For over 20 years, it was an open problem to prove completeness for MIN SOP-2-SOP, until Umans proved it in his dissertation [30], [31]. Moreover, he proved the $\Sigma_{2}^{P}$-completeness for a number of other logic minimization problems that model the most common operations performed in modern logic minimization packages (see ESPRESSO [3]), like maximal expansion and reduction of implicants.

Just as $N P$-complete problems probably require exponential time, $\Sigma_{2}^{P}=N P^{N P}$-complete problems probably require exponential time with access to an $N P$ oracle, so $\Sigma_{2}^{P}$-completeness allows us to classify what can or cannot be done in polynomial time with access to an $N P$ oracle. Notice that almost every heuristic, approximate, or exact method for solving logic minimization problems employs a tautology subroutine. If we assume the presence of such a subroutine, $\Sigma_{2}^{P}$-completeness plays exactly the role of $N P$-completeness for ordinary optimization problems: it distinguishes the intractable from the efficiently solvable.

We will now review the complexity of MIN SOP-2-SOP and of various logic minimization steps used in the major logic minimization suites when the input is given as an SOP.

\footnotetext{
${ }^{8}$ MIN SOP-2-SOP, called also MIN DNF in the computational complexity literature, is a specialized version of the problem MIN FORMULA, where the input is a generic Boolean formula. The latter problem motivated the definition of the polynomial hierarchy.

${ }^{9} \mathrm{~A}$ satisfiable DNF $\phi$ has an equivalent DNF of size 0 iff $\phi$ is a tautology, and one can check whether a DNF is satisfiable in polynomial time.
}

\section{A. Complexity of Term Expansion and Reduction}

The expand step in ESPRESSO attempts to expand as much as possible the set of minterms covered by a given term by removing literals from that term. One way to formalize the computational problem underlying this routine is as follows.

\section{SHORTEST IMPLICANT CORE}

INSTANCE: SOP formula $\phi=\vee_{i \in I} t_{i}, j \in I$, and an integer $k$.

QUESTION: Is there an implicant $t_{j}^{\prime}$ of $\phi$, with $\operatorname{lit}\left(t_{j}^{\prime}\right) \subseteq$ $\operatorname{lit}\left(t_{j}\right)$, of length at most $k$ ?

Theorem 6.1: SHORTEST IMPLICANT CORE is $\Sigma_{2}^{P}$ complete.

The proof is by reduction from 2-QBF and can be found in [30] and [31].

A variant of this problem asks not for a minimum length implicant $t_{j}^{\prime}$ for which $l i t\left(t_{j}^{\prime}\right) \subseteq l i t\left(t_{j}\right)$ but rather a minimal length (in other words, prime) implicant $t_{j}^{\prime}$ for which $\operatorname{lit}\left(t_{j}^{\prime}\right) \subseteq \operatorname{lit}\left(t_{j}\right)$. Usually, this latter variant is used in practice; in complexity terms, it is also "easier" since it lies in the class $P^{N P}$, as can be seen from the following algorithm. For each literal in term $t_{j}$, we use an $N P$ query to determine if $t_{j}$ with that literal deleted remains an implicant of $\phi$. If so, delete that literal and repeat from the resulting expanded term the process of deleting literals. Otherwise (no expanded term with a deleted literal is an implicant of $\phi$ ), the term is a prime. Overall, the running time is at most quadratic in the number of literals in $t_{j}$.

In a similar manner, we can formalize the step of reduce in ESPRESSO. This procedure attempts to reduce as much as possible a given term by adding literals while still covering the given function.

\section{LONGEST IMPLICANT EXTENSION (TERM REDUCTION)}

INSTANCE: An SOP formula $\phi=\vee_{i \in I} t_{i}$, a $j \in I$, and an integer $k$.

QUESTION: Is there an implicant $t_{j}^{\prime}$ of $\phi$, with $\operatorname{lit}\left(t_{j}^{\prime}\right) \supseteq$ $\operatorname{lit}\left(t_{j}\right)$, such that $\phi \equiv \vee_{i \in I \backslash\{j\}} t_{i} \vee t_{j}^{\prime}$ and $t_{j}^{\prime}$ has length of at least $k$ ?

It is noted in [30] and [31] that this problem is in the class $P_{\|}^{N P}$. This is true because $t_{j}^{\prime}$ can contain literal $\ell$ iff the conjunction $t_{j} \wedge \bar{\ell}$ is an implicant of $\vee_{i \in I \backslash\{j\}} t_{i}$. For each literal $\ell$, an $N P$ query will tell us whether the above condition holds. Thus, with polynomially many parallel $N P$ queries, we can determine exactly which literals can be added to $t_{j}$ to form $t_{j}^{\prime}$.

\section{B. Complexity of Minimum SOP}

This is the key problem introduced at the beginning of the section and asking, for a given a SOP formula $\phi$, what is the smallest SOP $\phi^{\prime}$ equivalent to $\phi$. The size of the SOP can be measured by the number either of literals or of terms, yielding, respectively, MIN LIT SOP-2-SOP Or MIN TERM SOP-2-SOP.

MIN LIT SOP-2-SOP

INSTANCE: An SOP formula $\phi$ and an integer $k$.

QUESTION: Is there an SOP $\phi^{\prime}$ with at most $k$ occurrences of literals and for which $\phi^{\prime} \equiv \phi$ ? 
Theorem 6.2: MIN LIT SOP-2-SOP is $\Sigma_{2}^{P}$-complete.

The proof is by reduction from SHORTEST IMPLICANT CORE and can be found in [30] and [31]. Given an instance $(\phi=$ $\left.\vee_{i \in I} t_{i}, j, k\right)$ of SHORTEST IMPLICANT CORE, the general idea of the reduction is to construct an SOP $\phi^{\prime}$ from $\phi$ in which every term except one corresponding to $t_{j}$ must occur in any equivalent SOP. The size of the minimum SOP equivalent to $\phi^{\prime}$ is then determined by the size of the smallest implicant core contained in that term.

As noted in [30] and [31], the proof also shows that a seemingly easier SOP minimization problem, which requires only minimality term-by-term, is also $\Sigma_{2}^{P}$-complete: given $\phi=$ $t_{1} \vee t_{2} \vee \ldots \vee t_{n}$ and a bound $k$, find an equivalent $\operatorname{SOP} \phi^{\prime}$, with at most $k$ occurrences of literals, of the form $\phi^{\prime}=t_{1}^{\prime} \vee$ $t_{2}^{\prime} \vee \ldots \vee t_{n}^{\prime}$ such that for all $i, \operatorname{lit}\left(t_{i}^{\prime}\right) \subseteq \operatorname{lit}\left(t_{i}\right)$.

The second main variant of MIN SOP-2-SOP asks for an equivalent SOP that is minimum with respect to terms (rather than occurrences of literals).

MIN TERM SOP-2-SOP

INSTANCE: An SOP formula $\phi$ and an integer $k$

QUESTION: Is there an SOP $\phi^{\prime}$ with at most $k$ terms and for which $\phi^{\prime} \equiv \phi ?$

Theorem 6.3: MIN TERM SOP-2-SOP is $\Sigma_{2}^{P}$-complete.

The proof can be found in [30, Th. 5.5, p. 44] by reduction from SHORTEST IMPLICANT CORE.

\section{Complexity of Irredundant Cover}

The following problem formalizes the step of irredundant in ESPRESSO, namely, remove as many terms as possible from a given SOP.

\section{IRREDUNDANT COVER}

INSTANCE: An SOP formula $\phi=\vee_{i \in I} t_{i}$ and an integer $k$. QUESTION: Is there a subset $I^{\prime} \subseteq I$ such that $\phi \equiv \vee_{i \in I^{\prime}} t_{i}$ and $\left|I^{\prime}\right| \leq k$ ?

Theorem 6.4: IRREDUNDANT COVER is $\Sigma_{2}^{P}$-complete.

The proof can be found in [30, Th. 5.7, p. 50] by reduction from SHORTEST IMPLICANT CORE.

\section{Complexity of Detecting Implicants}

The most basic logic containment operation is to check if a conjunction $t$ is an implicant of $\operatorname{SOP} \phi$.

IMPLICANT

INSTANCE: An SOP formula $\phi$ and a term $t$.

QUESTION: Is $t$ an implicant of $\phi$ ?

Theorem 6.5: IMPLICANT is $c o N P$-complete.

Proof: This problem is in $c o N P$ because the complement problem of determining whether $t$ is not an implicant of $\phi$ can be solved by guessing a point covered by $t$ but not $\phi$.

We show that it is $c o N P$-complete by reducing from SOPVALIDITY. Given an SOP $\phi$, which is an instance of SOPVALIDITY, we produce the instance $(\phi, 1)$ of IMPLICANT. It is clear that 1 is an implicant of $\phi$ iff $\phi$ is a tautology.

The next problem formalizes the step of detecting essential implicants. Here, we want to determine if a term $t_{j}$ of an SOP $\phi$ covers some point that no other term in $\phi$ covers.
ESSENTIAL IMPLICANT

INSTANCE: An SOP formula $\phi=\vee_{i \in I} t_{i}$ and $j \in I$.

QUESTION: Is $\phi \not \equiv \phi^{\prime}$, where $\phi^{\prime}=\vee_{i \in I \backslash\{j\}} t_{i}$ ?

Theorem 6.6: ESSENTIAL IMPLICANT is $N P$-complete.

Proof: The problem is in $N P$ because one can guess a point and verify that it is covered by $t_{j}$ but is not covered by $\vee_{i \in I \backslash\{j\}} t_{i}$ (note that checking whether a given point is covered by a term is a polynomial-time operation).

Completeness is shown by reduction from CNF-SAT. Let $\gamma\left(x_{1}, \ldots, x_{n}\right)$ be an instance of CNF-SAT. Introduce a new variable $z$ and construct the SOP formula $\phi=z \vee(z \wedge \neg \gamma)$. Set $j$ so that $t_{j}$ is the term $z$ in this SOP.

We claim that $\gamma$ is satisfiable iff $z$ is essential (i.e., $\phi \not \equiv$ $\left.\phi^{\prime} \equiv(z \wedge \neg \gamma)\right)$. If $\gamma$ is satisfiable, then there exists some $\left(a_{1}, \ldots, a_{n}\right)$ for which $\gamma\left(a_{1}, \ldots, a_{n}\right)=1$; this implies that $(z \wedge \neg \gamma)$ does not cover the extension of this point that sets $z=1$, while $z$ clearly does cover it. This means that $z$ is essential. In the other direction, if $\gamma$ is not satisfiable, then $(z \wedge \neg \gamma)$ is equivalent to $z$, and so $z$ is not essential.

Another basic operation is that of detecting prime implicants. PRIME IMPLICANT

INSTANCE: An SOP formula $\phi$ and a term $t$.

QUESTION: Is $t$ a prime implicant of $\phi$ ?

Theorem 6.7: PRIME IMPLICANT is $D P$-complete.

Proof: It is in $D P$ because $t$ is a prime implicant iff $t$ is an implicant (a problem in $c o N P$ as noted above), and every shortening of $t$ by deleting one literal is not an implicant (a problem in $N P$ ).

We show that it is $D P$-hard by reduction from SAT-UNSAT. Let $\left(\phi, \phi^{\prime}\right)$ be an instance of SAT-UNSAT (both $\phi$ and $\phi^{\prime}$ are CNFs). We produce the following $\operatorname{SOP}(z$ is a fresh variable): $\phi^{\prime \prime} \equiv(\neg z \wedge \neg \phi) \vee\left(z \wedge \neg \phi^{\prime}\right)$. Our instance of PRIME IMPLICANT is $\left(\phi^{\prime \prime}, z\right)$.

We claim that $z$ is a prime implicant of $\phi^{\prime \prime}$ iff $\phi$ is satisfiable and $\phi^{\prime}$ is unsatisfiable. If $\phi^{\prime}$ is unsatisfiable, then it is clear that $z$ is an implicant of $\phi^{\prime \prime}$; at the same time, if $\phi$ is satisfiable, then $z$ must be prime, because 1 is not an implicant of $\phi^{\prime \prime}$. In the other direction, if $z$ is an implicant of $\phi^{\prime \prime}$, then $\phi^{\prime}$ must be unsatisfiable; and if $z$ is also prime, then 1 cannot be an implicant of $\phi^{\prime \prime}$, which implies that $\phi$ must be satisfiable.

\section{E. Complexity of Shortest Implicant}

A relaxation of SHORTEST IMPLICANT CORE is to look for a shortest implicant of SOP $\phi$, not necessarily obtained by expanding a term of $\phi$.

\section{SHORTEST IMPLICANT}

INSTANCE: An SOP formula $\phi=\vee_{i \in I} t_{i}$ and an integer $k$. QUESTION: Is there an implicant $t_{j}^{\prime}$ of $\phi$ of length at most $k$ ?

One might expect that this problem is $\Sigma_{2}^{P}$-complete too since it is in $\Sigma_{2}^{P}$, like the others. However, it turns out that it is likely easier than $\Sigma_{2}^{P}$-complete by exploiting the following fact, proved by Umans [30], [31]: any shortest implicant of $\phi$ may be obtained from some term $t_{i}$ of $\phi$ with at most $\log n$ additions of literals and at $\operatorname{most} \log n$ deletions of literals. As a consequence to decide SHORTEST IMPLICANT, it requires only 
$O\left(\log ^{2} n\right)$-limited nondeterminism and a single $\operatorname{coNP}$ query. Given an instance $\left(\phi=t_{1} \wedge t_{2} \wedge \ldots \wedge t_{n}, k\right)$, one guesses an index $i$, a set $I$ of $\log n$ literals to add, a set $D$ of $\log n$ literals to delete, and constructs a candidate conjunction $C=$ $\left(t_{i} \cup I\right) \backslash D$. We answer "yes" if $C$ is well formed, has length of at most $k$, and a query to a $c o N P$ oracle indicates that it is an implicant of $\phi$.

Moreover, Umans showed [30], [31] that this problem is in fact complete for a new complexity class including the problems solvable by $O\left(\log ^{2} n\right)$ limited nondeterminism and a single interaction with a $\operatorname{coNP}$ oracle, denoted by $\mathrm{GC}\left(\log ^{2} n, \operatorname{coN} P\right)$ and lying between $\operatorname{coN} P$ and $\Sigma_{2}^{P}$. This result indicates that while the problem is unlikely to be $\Sigma_{2}^{p}$ complete, it is also unlikely to be any "easier" than the above algorithm indicates; in particular, it is not likely to be in $\operatorname{coN} P$.

Theorem 6.8: SHORTEST IMPLICANT is $\mathrm{GC}\left(\log ^{2} n\right.$, coNP)-complete.

The formula and circuit versions of SHORTEST IMPLICANT instead are $\Sigma_{2}^{P}$-complete (see again [30] and [31]).

\section{F. Complexity of Complementation}

In ESPRESSO, given an SOP cover of the offset and dcset, an SOP cover of the offset is computed, because the maximal expansion of a cube is obtained by checking that it does not intersect any cube of the offset. Therefore, given an SOP $\phi$, it is important to compute a small SOP representation of the complement of $\phi$. This problem is captured as follows.

MIN SOP COMPLEMENT

INSTANCE: An SOP formula $\phi$ and an integer $k$ in unary (i.e., $k$ is given in the input by a string of $k$ ones).

QUESTION: Is there an SOP formula $\phi^{\prime}$ with at most $k$ occurrences of literals for which $\phi^{\prime} \equiv \neg \phi$ ?

Note that if $k$ were represented in binary, then we would not readily be able to show that the problem is even in $\Sigma_{2}^{P}$ since there are SOP formulas $\phi$ whose complement requires exponential size in $|\phi|$. However, we avoid this difficulty if $k$ is represented in unary. In that case, the input has size of at least $k$ and then the problem lies in $\Sigma_{2}^{P}$ since any potential $\phi^{\prime}$ has size of only polynomial in the size of the input.

In fact, Schaefer and Umans [23] show that this problem is $\Sigma_{2}^{P}$-complete.

Theorem 6.9: MIN SOP COMPLEMENT is $\Sigma_{2}^{P}$-complete.

Proof: In [23], they show that a problem called SHORT $\mathrm{CNF}$ is $\Sigma_{2}^{P}$-complete. In this problem, the input is an SOP formula $\phi$ and an integer $k$ represented in unary; the question is whether there is a CNF formula $\phi^{\prime}$ with at most $k$ occurrences of literals for which $\phi^{\prime} \equiv \phi$. It is clear that this occurs iff there is an SOP formula $\phi^{\prime \prime}$ with at most $k$ occurrences of literals for which $\phi^{\prime \prime} \equiv \neg \phi$, since the negation of an SOP formula $\phi^{\prime \prime}$ can be represented by a CNF formula of the same size, and vice versa.

\section{G. Complexity of State Assignment}

A finite state machine (or "finite state transducer") is specified by a transition function $\delta: Q \times\{0,1\}^{s} \rightarrow Q \times\{0,1\}^{t}$, where $Q$ is a finite set of states, and $\{0,1\}^{s}$ is the set of inputs and $\{0,1\}^{t}$ is the set of outputs. Equivalently, we may specify the transition function with a function

$$
f: Q \times\{0,1\}^{s} \times Q \times\{0,1\}^{t} \rightarrow\{0,1\}
$$

with the property that $f\left(q, i, q^{\prime}, o\right)=1$ iff $\delta(q, i)=\left(q^{\prime}, o\right)$. It is desirable to express $f$ as a small SOP. To do this, we need a state encoding function $e: Q \rightarrow\{0,1\}^{m}$. Then we may ask for a minimum SOP $\phi$ representing the function $f_{e}$ defined by ${ }^{10}$

$$
f_{e}\left(e(q), i, e\left(q^{\prime}\right), o\right)=1 \quad \Leftrightarrow \quad f\left(q, i, q^{\prime}, o\right)=1 .
$$

The decision problem associated with this optimization is as follows.

STATE ASSIGNMENT

INSTANCE: An SOP $\phi$ representation of a function $f_{e}$ specifying a finite state machine with states $Q$, and an integer $k$.

QUESTION: Is there an integer $m^{\prime}$ and an encoding function $e^{\prime}: Q \rightarrow\{0,1\}^{m^{\prime}}$ for which $f_{e^{\prime}}$ has an SOP representation $\phi^{\prime}$ with at most $k$ terms ${ }^{11}$ ?

It had been shown by Keutzer and Richards [14] that STATE ASSIGNMENT is $c o N P$-hard (lower bound) and contained in $\Sigma_{2}^{p}$ (upper bound). A complete account of the early results on the complexity of this problem is available in [32]. In the degenerate case in which there is only a single state, STATE ASSIGNMENT becomes MIN TERM SOP-2-SOP. We thus obtain the new result.

Theorem 6.10: STATE ASSIGNMENT is $\Sigma_{2}^{P}$-complete.

The practitioner may protest that lumping together two-level minimization and state assignment in the same complexity classes is highly unsatisfactory since the latter problem is much harder than the former in practice. This is but one example where the coarse classification afforded by the classes of the $\mathrm{PH}$ would benefit from some refinement that captures the observed difference in the difficulty of problems within the same class. The classification of $N P$-complete problems according to their approximability is an example of such a refinement; however, here, it seems that approximability would not separate, e.g., MIN SOP-2-SOP from STATE ASSIGNMENT, as MIN SOP-2-SOP is already extremely hard to approximate [29], [30].

\section{CONCLUSION}

Two-level logic minimization is the quintessential problem in digital design automation. In this paper, we have presented a consistent account of the computational complexity of decision problems capturing two-level logic minimization. We hope that this provides a coherent picture of results scattered in many sources and fills a few gaps in the older literature.

\footnotetext{
${ }^{10}$ This is the relational representation of a finite state machine; alternatively, one may use a functional representation by means of a multiple-output function whose outputs correspond to all the encoded next state and external output variables.

${ }^{11}$ We can also consider the variant in which $\phi^{\prime}$ must have at most $k$ literals.
} 


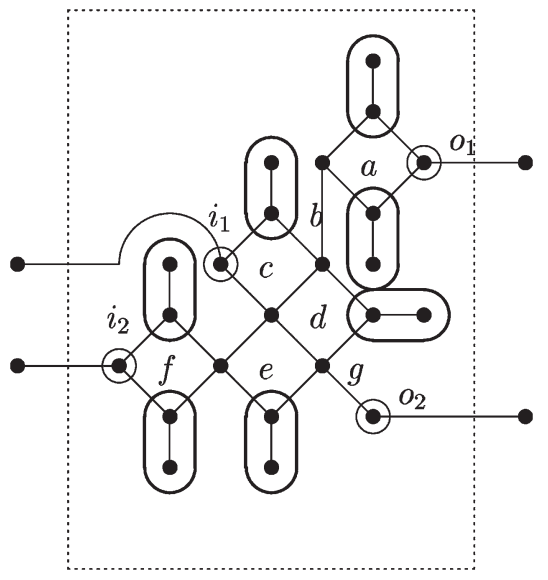

Fig. 9. AND/OR gadget with essential terms in cover.

The major points to take away are the following.

- When the function is explicitly described by its onset and offset (or even just its onset), the problem of finding a minimum SOP representation is NP-complete. This problem is essentially a covering problem, and $N P$ completeness follows by either the Gimpel reduction in Section IV or the more involved Masek reduction in Section V.

- When the function is implicitly described by an SOP form, the complexity of finding a minimum SOP representation is $\Sigma_{2}^{P}$-complete. This means that (subject to accepted assumptions from computational complexity) no polynomial-time algorithm can solve this problem even when charging only a single time step for every call to a TAUTOLOGY subroutine.

- When the function is implicitly described by an SOP form, many of the subproblems used in the major logic minimization suites (i.e., ESPRESSO) turn out to lie between $N P$ and $\Sigma_{2}^{P}$, and several are $\Sigma_{2}^{p}$-complete.

In closing, we mention two very natural problems whose complexity remains open.

\section{MIN CIRCUIT-2-CIRCUIT}

INSTANCE: A Boolean circuit $C$ and an integer $k$.

QUESTION: Is there a Boolean circuit $C^{\prime}$ of size at most $k$ that computes the same function as $C$ ?

This problem is in $\Sigma_{2}^{P}$ and conjectured to be $\Sigma_{2}^{P}$-complete. In fact, even when $C$ and $C^{\prime}$ are required to be circuits of depth 3 (i.e., sum-of-products-of-sums or product-of-sums-ofproducts), no proof of $\Sigma_{2}^{P}$-completeness is known.

The "explicit" version of this problem is as follows.

\section{FULL TRUTH TABLE MIN CIRCUIT}

INSTANCE: Onset and offset of a completely specified function $f$ and an integer $k$.

QUESTION: Is there a Boolean circuit $C$ of size at most $k$ that computes $f$ ?

This problem is in $N P$, and it is not known whether it is $N P$ complete or not. This problem has some interesting connections to other questions in computational complexity that have been outlined in [12].

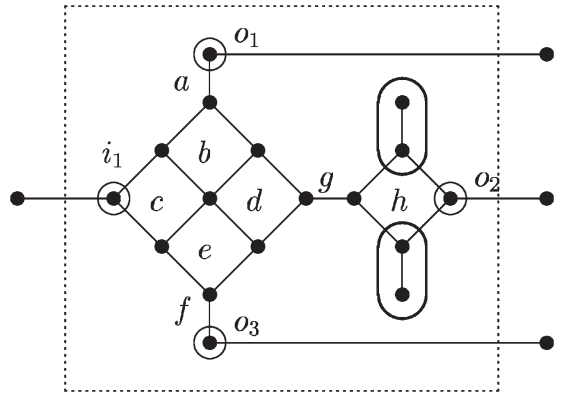

Fig. 10. GENERATOR gadget with essential terms in cover.

The complexity of minimization problems represented with decision diagrams falls beyond the scope of this paper. We refer the interested reader to [11] and [25] as starting points.

\section{APPENDIX \\ Case Analyses}

Proof of Lemma 5.1: We refer to Fig. 9. The seven terms that are essential in any cover are pictured, and the possible other terms are labeled $a, b, c, d, e, f, g$. The following table describes an optimal cover for each possible truth assignment to the four I/O nodes $i_{1}, i_{2}, o_{1}$, and $o_{2}$, i.e.,

\begin{tabular}{l|l|l|l|l|l}
\hline$i_{1}$ & $i_{2}$ & $o_{1}$ & $o_{2}$ & 7 essential, plus & total \\
\hline false & false & false & false & $\{f, c, a, g\}$ & $\mathbf{1 1}$ \\
false & false & false & true & $\{f, c, a, g\}$ & 11.5 \\
false & false & true & false & $\{f, c, b, g\}$ & 11.5 \\
false & false & true & true & $\{f, c, b, e\}$ & 12 \\
\hline false & true & false & false & $\{c, a, g, e\}$ & 11.5 \\
false & true & false & true & $\{c, a, e\}$ & $\mathbf{1 1}$ \\
false & true & true & false & $\{c, g, b, e\}$ & 12 \\
false & true & true & true & $\{c, b, e\}$ & 11.5 \\
\hline true & false & false & false & $\{f, a, g, c\}$ & 11.5 \\
true & false & false & true & $\{f, a, d\}$ & $\mathbf{1 1}$ \\
true & false & true & false & $\{f, g, b, c\}$ & 12 \\
true & false & true & true & $\{f, b, e\}$ & 11.5 \\
\hline true & true & false & false & $\{a, g, c, f\}$ & 12 \\
true & true & false & true & $\{a, b, e\}$ & 11.5 \\
true & true & true & false & $\{g, b, e\}$ & 11.5 \\
true & true & true & true & $\{b, e\}$ & $\mathbf{1 1}$ \\
\hline
\end{tabular}

Proof of Lemma 5.2: We refer to Fig. 10. The two terms that are essential in any cover are pictured, and the possible other terms are labeled $a, b, c, d, e, f, g, h$. The following table 
describes an optimal cover for each possible truth assignment to the four $\mathrm{I} / \mathrm{O}$ nodes $i_{1}, o_{1}, o_{2}$, and $o_{3}$. The following table describes an optimal cover for each possible truth assignment to the four I/O nodes, i.e.,

\begin{tabular}{l|l|l|l|l|l}
\hline$i_{1}$ & $o_{1}$ & $o_{2}$ & $o_{3}$ & 2 essential, plus & total \\
\hline false & false & false & false & $\{a, c, f, h, d\}$ & 7 \\
false & false & false & true & $\{a, c, h, d, f\}$ & 7.5 \\
false & false & true & false & $\{a, c, f, d, g\}$ & 7.5 \\
false & false & true & true & $\{a, c, e, d, g\}$ & 8 \\
false & true & false & false & $\{c, f, h, d, b\}$ & 7.5 \\
false & true & false & true & $\{c, h, d, b, e\}$ & 8 \\
false & true & true & false & $\{c, f, g, b, e\}$ & 8 \\
false & true & true & true & $\{c, g, b, e\}$ & 7.5 \\
\hline true & false & false & false & $\{a, f, h, d, c\}$ & 7.5 \\
true & false & false & true & $\{a, h, e, b, d\}$ & 8 \\
true & false & true & false & $\{a, f, g, c, d\}$ & 8 \\
true & false & true & true & $\{a, g, b, e\}$ & 7.5 \\
true & true & false & false & $\{h, f, b, c, d\}$ & 8 \\
true & true & false & true & $\{h, b, d, e\}$ & 7.5 \\
true & true & true & false & $\{f, b, e, g\}$ & 7.5 \\
true & true & true & true & $\{g, b, e\}$ & 7 \\
\hline
\end{tabular}

Proof of Lemma 5.4: By construction, every pair of adjacent points in the adjacency diagram is embedded as a pair of adjacent hypercube nodes. Now consider two nonadjacent points in the adjacency diagram. We argue that they are embedded as nonadjacent hypercube nodes. There are several cases, enumerated below. It will be helpful to refer to the three segments of a wire, where segment $s$ consists of the point numbered $s$ in the list just before Lemma 5.4 and all of the points on a shortest path to the point numbered $s+1$, except point $s+1$ itself.

1) The two points belong to different AND/OR or GENERATOR gadgets. The fact that the gadget IDs are even parity ensures that these two points are embedded at vectors that differ in at least two positions in the first field.

2) One point belongs to an AND/OR or GENERATOR gadget, and the other point belongs to a wire attached to that gadget. If the wire point is not adjacent to one of the wire endpoints, then it is embedded at a vector that has weight at least two in the third field, and so it differs from all of the vectors at which gadget points are embedded in at least two positions. If the wire point is one of the two wire points adjacent to a wire endpoint, then it is embedded at a vector that has weight one in the third field. This vector is adjacent to exactly one vector at which a gadget point is embedded (since that vector must match exactly in the first two fields), and this vector is the wire endpoint.
3) One point belongs to an AND/OR or GENERATOR gadget, and the other belongs to a wire not attached to that gadget. If the wire point lies in segment 1 or 3 , then these two points are embedded at vectors that differ by at least two positions in the first field. If the wire point lies in segment 2, then these two points are embedded at vectors that differ in at least two positions in the third field.

4) The two points belong to the same wire (with ID id $(k)$ ) connecting $(\operatorname{id}(i), x, \overrightarrow{0})$ to $(\operatorname{id}(j), y, \overrightarrow{0})$. If they lie in the same wire segment, then our choice of a shortest path between the segment endpoints ensures that the two points are embedded at vectors that differ in at least two positions. If the two points are from different segments, then we consider three subcases.

a) One point lies in segment 1 and the other lies in segment 2 . Then the first point is embedded at a vector that differs from $(\operatorname{id}(i), x, \operatorname{id}(k))$ in at least one position in the third field and the second point is embedded at a vector that differs from $(\operatorname{id}(i), x, \operatorname{id}(k))$ in at least one position in the first two fields. Altogether, the two points are embedded at vectors that differ in at least two positions.

b) One point lies in segment 2 and the other lies in segment 3 . Then the first point is embedded at a vector that differs from $(\operatorname{id}(j), y, \operatorname{id}(k))$ in at least one position in the first two fields, and the second point is embedded at a vector that differs from $(\operatorname{id}(j), y, \operatorname{id}(k))$ in at least one position in the third field. Altogether, the two points are embedded at vectors that differ in at least two positions.

c) One point lies in segment 1 and the other lies in segment 3 . Then the two points are embedded at vectors that differ by at least two positions in the first field.

5) The two points belong to different wires; the first wire (with $\operatorname{ID} \operatorname{id}(k)$ ) connects $(\operatorname{id}(i), x, \overrightarrow{0})$ to $(\operatorname{id}(j), y, \overrightarrow{0})$, and the second wire (with $\left.\operatorname{ID} \operatorname{id}\left(k^{\prime}\right)\right)$ connects $\left(\operatorname{id}\left(i^{\prime}\right), x^{\prime}, \overrightarrow{0}\right)$ to $\left(\operatorname{id}\left(j^{\prime}\right), y^{\prime}, \overrightarrow{0}\right)$. There are several subcases, depending on the segment in which each of the two wire points lies.

a) The first point lies in segment 1 and the second point lies in segment 1 . Then either the two points are embedded at vectors that differ in at least two positions in the first field, or else $i=i^{\prime}$ and then $x$ and $x^{\prime}$ differ in at least two positions since $\mathrm{I} / \mathrm{O}$ nodes within a gadget differ in at least two positions.

b) The first point lies in segment 2 and the second point lies in segment 2 . Then the two points are embedded at vectors that differ in at least two positions in the third field (since $k \neq k^{\prime}$ ).

c) The first point lies in segment 3 and the second point lies in segment 3 . Then either the two points are embedded at vectors that differ in at least two positions in the first field, or else $j=j^{\prime}$ and then $y$ and $y^{\prime}$ differ in at least two positions since $\mathrm{I} / \mathrm{O}$ nodes within a gadget differ in at least two positions.

d) The first point lies in segment 1 and the second point lies in segment 3 . Then either the two points are embedded at vectors that differ in at least two positions in the first field, or else $i=j^{\prime}$ and then $x$ and $y^{\prime}$ differ in 
at least two positions since I/O nodes within a gadget differ in at least two positions.

e) The first point lies in segment 3 and the second point lies in segment 1 . Then either the two points are embedded at vectors that differ in at least two positions in the first field, or else $j=i^{\prime}$ and then $y$ and $x^{\prime}$ differ in at least two positions since I/O nodes within a gadget differ in at least two positions.

f) The first point lies in segment 1 and the second point lies in segment 2 . Then the point in segment 1 is embedded at a vector whose third field either has weight $<2 \ell-1$ or else it differs from $\operatorname{id}(k)$ in only one position. In the latter case, its third field must differ from id $\left(k^{\prime}\right)$ in at least three positions since id $(k)$ and $\operatorname{id}\left(k^{\prime}\right)$ differ in at least four positions.

g) The first point lies in segment 2 and the second point lies in segment 1 . This case is the same as the previous with the roles of $k$ and $k^{\prime}$ interchanged.

h) The first point lies in segment 2 and the second point lies in segment 3 . Then the point in segment 3 is embedded at a vector whose third field either has weight $<2 \ell-1$ or else it differs from id $\left(k^{\prime}\right)$ in only one position. In the latter case, its third field must differ from $\operatorname{id}(k)$ in at least three positions since id $(k)$ and $\operatorname{id}\left(k^{\prime}\right)$ differ in at least four positions.

i) The first point lies in segment 3 and the second point lies in segment 2 . This case is the same as the previous with the roles of $k$ and $k^{\prime}$ interchanged.

This establishes that the adjacency diagram is properly realized with the embedding we have described, and in particular, no unintended adjacencies have been introduced.

Note Added in Proof: Feldman [33] has recently obtained an alternative proof of Masek's result, together with inapproximability results. Also, some results on the complexity of implicant finding have been obtained independently by Goldsmith et al. [34].

\section{ACKNOWLEDGMENT}

The authors thank D. S. Johnson for kindly providing a hardcopy of Masek's technical report. T. Villa thanks R. Brayton, $\mathrm{K}$. Keutzer, and A. Oliveira for related discussions.

\section{REFERENCES}

[1] E. Allender, L. Hellerstein, P. M. McCabe, T. Pitassi, and M. Saks, "Minimizing DNF formulas and AC0 circuits given a truth table," ECCC Tech. Rep. TR05-126, 2005.

[2] D. Bovet and P. Crescenzi, Introduction to the Theory of Complexity. Englewood Cliffs, NJ: Prentice-Hall, 1994.

[3] R. Brayton, G. Hachtel, C. McMullen, and A. Sangiovanni-Vincentelli, Logic Minimization Algorithms for VLSI Synthesis. Boston, MA: Kluwer, 1984.

[4] R. Brayton, A. Sangiovanni-Vincentelli, and G. Hachtel, "Multi-level logic synthesis," Proc. IEEE, vol. 78, no. 2, pp. 264-300, Feb. 1990.

[5] V. Ciriani, "Synthesis of SPP three-level logic networks using affine spaces," IEEE Trans. Comput.-Aided Des. Integr. Circuits Syst., vol. 22, no. 10 , pp. 1310-1323, Oct. 2003.

[6] O. Coudert, "Two-level logic minimization: An overview," Integration, vol. 17, no. 2, pp. 97-140, Oct. 1994.

[7] S. L. A. Czort, "The complexity of minimizing disjunctive normal form formulas," M.S. thesis, Dept. Comput. Sci., Univ. Aarhus, Aarhus, Denmark, 1999
[8] M. Gao, J.-H. Jiang, Y. Jiang, Y. Li, A. Mishchenko, S. Sinha, T. Villa, and R. Brayton, "Optimization of multi-valued multi-level networks," in Proc. Int. Symp. Multiple-Valued Logic, Sendai, Japan, May 2002, pp. $168-177$.

[9] M. R. Garey and D. S. Johnson, Computers and Intractability: A Guide to the Theory of NP-Completeness. San Francisco, CA: Freeman, 1979.

[10] J. Gimpel, "A method of producing a Boolean function having an arbitrarily prescribed prime implicant table," IRE Trans. Electron. Comput., vol. EC-14, no. 6, pp. 485-488, Jun. 1965.

[11] K. Hayase and H. Imai, "OBDDs of a monotone function and of its prime implicants," in Proc. 7th Int. Symp. Algorithms and Computation (ISAAC), Osaka, Japan, 1178, Lecture Notes in Computer Science. New York: Springer-Verlag, 1996, pp. 136-145.

[12] V. Kabanets and J.-Y. Cai, "Circuit minimization problem," in Proc. 32nd Anпи. ACM Symp. Theory Computing (STOC), Portland, OR, 2000, pp. 73-79.

[13] R. Karp, "Reducibility among combinatorial problems," in Complexity of Computer Computations, R. E. Miller and J. W. Thatcher, Eds. New York: Plenum, 1972, pp. 85-103.

[14] K. Keutzer and D. Richards, "Computational complexity of logic synthesis and optimization," presented at the Int. Workshop Logic Synthesis, Research Triangle Park, NC, May 1989.

[15] W. J. Masek, "Some NP-complete set covering problems," M.S. thesis, MIT Lab. Comput. Sci., Massachusetts Inst. Technol., Cambridge, May 1978.

[16] E. McCluskey, "Minimization of Boolean functions," Bell Lab. Tech. J., vol. 35, no. 6, pp. 1417-1444, Nov. 1956.

[17] P. McGeer, J. Sanghavi, R. Brayton, and A. Sangiovanni-Vincentelli, "ESPRESSO-SIGNATURE: A new exact minimizer for logic functions," IEEE Trans. Very Large Scale Integr. (VLSI) Syst., vol. 1, no. 4, pp. 432440, Dec. 1993.

[18] F. Mo and R. Brayton, "PLA-based regular structures and their synthesis," IEEE Trans. Comput.-Aided Des. Integr. Circuits Syst., vol. 22, no. 6, pp. 723-729, Jun. 2003.

[19] C. Papadimitriou, Computational Complexity. Reading, MA: AddisonWesley, 1994.

[20] W. Paul, "Boolean minimum polynomials and covering problems," (in German) Acta Inform., vol. 4, no. 4, pp. 321-336, 1975, Transl.: Boolesche Minimalpolynome and Ueberdeckungsprobleme.

[21] R. Rudell and A. Sangiovanni-Vincentelli, "Multiple-valued minimization for PLA optimization," IEEE Trans. Comput.-Aided Des. Integr. Circuits Syst., vol. CAD-6, no. 5, pp. 727-750, Sep. 1987.

[22] S. Sapra, M. Theobald, and E. M. Clarke, "Sat-based algorithms for logic minimization," in Proc. Int. Conf. Computer Design, San Jose, CA, Oct. 2003, p. 510.

[23] M. Schaefer and C. Umans, "Completeness in the polynomial-time hierarchy: A compendium," SIGACT News, vol. 33, no. 3, pp. 32-49, Sep. 2002.

[24] - , "Completeness in the polynomial-time hierarchy: Part II," SIGACT News, vol. 33, no. 4, pp. 22-36, Dec. 2002

[25] D. Sieling, "The complexity of minimizing and learning OBDDs and FBDDs," Discrete Appl. Math., vol. 122, no. 1-3, pp. 263-282, Oct. 2002.

[26] L. J. Stockmeyer, "The polynomial-time hierarchy," Theor. Comput. Sci., vol. 3, no. 1, pp. 1-22, Oct. 1976.

[27] T. Strzemecki, "Polynomial-time algorithms for the generation of prime implicants," J. Complex., vol. 8, no. 1, pp. 37-63, Mar. 1992.

[28] M. Theobald and S. Nowick, "Fast heuristic and exact algorithms for two-level hazard-free logic minimization," IEEE Trans. Comput.-Aided Des. Integr. Circuits Syst., vol. 17, no. 11, pp. 1130-1147, Nov. 1998.

[29] C. Umans, "Hardness of approximating $\Sigma_{2}^{P}$ minimization problems," in Proc. 40th Annu. IEEE Symp. Foundations Computer Science (FOCS), New York, 1999, pp. 465-474.

[30] _ - "Approximability and completeness in the polynomial hierarchy," Ph.D. dissertation, Dept. Elect., Eng. Comput. Sci., Univ. California, Berkeley, 2000.

[31] _ - "The minimum equivalent DNF problem and shortest implicants," J. Comput. Syst. Sci., vol. 63, no. 4, pp. 597-611, Dec. 2001.

[32] T. Villa, T. Kam, R. Brayton, and A. Sangiovanni-Vincentelli, Synthesis of FSMs: Logic Optimization. Norwell, MA: Kluwer, 1997.

[33] V. Feldman, "Hardness of approximate two-level logic minimization and PAC learning with membership queries," ECCC, Potsdam-Babelsberg, Germany, Tech. Rep. TR05-127, 2005.

[34] J. Goldsmith, M. Hagen, and M. Mundhenk, "Complexity of DNF and isomorphism of monotone formulas," in Proc. 30th Int. Symp. Mathematical Foundations Computer Science (MFCS), Gdansk, Poland, Aug. 2005, pp. $410-421$. 


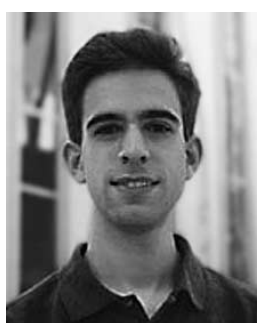

Christopher Umans received the undergraduate degree in computer science and mathematics from Williams College, Williamstown, MA, in 1996 and the Ph.D. degree in computer science from the University of California, Berkeley, in 2000.

From 2000 to 2002, he was a Post-Doc in the theory group at Microsoft Research, Redmond, WA. Since 2002, he has been an Assistant Professor of Computer Science at the California Institute of Technology, Pasadena. His research interests lie in theoretical computer science, especially computational complexity theory. He has worked on problems in complexity, derandomization, explicit combinatorial constructions, hardness of approximation, and algorithms.

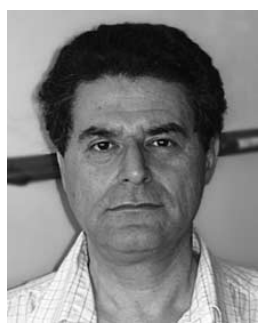

Tiziano Villa received the B.S. degree in mathematics from the University of Milan, Milan, Italy, in 1977, took the Mathematical Tripos, Part III, at the University of Cambridge, Cambridge, U.K., in 1982, and received the M.S. degree in computer science and the Ph.D. degree in electrical engineering from the University of California, Berkeley, in 1987 and 1995 , respectively.

From 1980 to 1985 , he was a Computer-Aided Design Specialist at the Integrated Circuits Division, CSELT Labs, Torino, Italy, and then from 1986 to 1996 was a Research Assistant at the Electronics Research Laboratory, University of California, Berkeley. In 1997, he was a Research Scientist at the PARADES Labs, Rome, Italy. Since 2002, he has been an Associate Professor at the Department of Electrical, Industrial and Mechanical Engineering (DIEGM), Universita' di Udine, Italy. He coauthored the books Synthesis of FSMs: Functional Optimization (Kluwer, 1997) and Synthesis of FSMs: Logic Optimization (Kluwer, 1997). His research interests include logic synthesis, formal verification, combinatorial optimization, automata theory, and hybrid systems. His contributions are mainly in the areas of combinational and sequential logic synthesis.

Dr. Villa was awarded the Tong Leong Lim Pre-Doctoral Prize by the EECS Department, University of California, Berkeley, in May 1991.

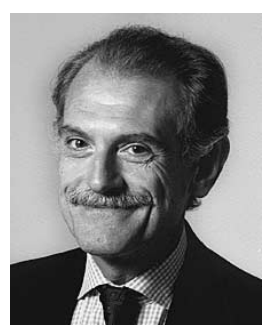

Alberto L. Sangiovanni-Vincentelli (M'74SM'81-F'83) received the "Dottore in Ingegneria" degree (summa cum laude) in electrical engineering and computer science from the Politecnico di Milano, Milan Italy, in 1971.

Since 1976, he has been on the Faculty of the University of California, Berkeley, and currently holds the Edgar L. and Harold H. Buttner Chair of Electrical Engineering and Computer Sciences. From 1980 to 1981 , he was a Visiting Scientist at the Mathematical Sciences Department, IBM T. J. Watson Research Center. In 1987, he was Visiting Professor at Massachusette Institute of Technology. He has held a number of Visiting Professor positions at Italian Universities, including Politecnico di Torino, Universitá di Roma, La Sapienza, Universitá di Roma, Tor Vergata, Universitá di Pavia, Universitá di Pisa, Scuola di Sant' Ann. He was a Co-Founder of Cadence and Synopsys, the two leading companies in the area of electronic design automation. He is the Chief Technology Adviser of Cadence. He is a member of the Board of Directors of Cadence, UPEK, Sonics, Gradient and Accent, an ST Microelectronics-Cadence joint venture he helped founding. He is a member of the HP Strategic Technology Advisory Board and of the Science and Technology Advisory Board of General Motors. He is the Founder and Scientific Director of PARADES, a European Group of Economic Interest supported by Cadence, Magneti-Marelli, and ST Microelectronics. He is a member of the High-Level Group of the EU Artemis Platform. He is the author of over 650 papers and 15 books in the area of design tools and methodologies, large-scale systems, embedded controllers, hybrid systems, and innovation.

Dr. Sangiovanni-Vincentelli is a member of the National Academy of Engineering since 1998. In 1981, he received the Distinguished Teaching Award of the University of California. He has received numerous research awards including the Guillemin-Cauer Award (1982-1983), the Darlington Award (1987-1988) of the IEEE for the best paper bridging theory and applications, two awards for the best paper published in the Transactions on CAS and $\mathrm{CAD}$, and three best paper awards and one best presentation award at the Design Automation Conference. He received the worldwide 1995 Graduate Teaching Award of the IEEE (a technical field award for inspirational teaching of graduate students). In 2002, he was the recipient of the Aristotle Award of the Semiconductor Research Corporation. In 2001, he was given the prestigious Kaufman Award of the Electronic Design Automation Council for pioneering contributions to EDA. 